\title{
Cavitation Erosion of Ni-Based Superalloys Manufactured by Forging and Additive Manufacturing
}

\author{
Gabriel Taillon (D) Kazuyoshi Miyagawa
}

Submitted: 10 May 2021/in revised form: 24 July 2021 / Accepted: 2 September 2021/Published online: 5 October 2021

(C) ASM International 2021

\begin{abstract}
Inconel pipes that transport cryogenic fluids in rocket engines manufactured by additive manufacturing (AM) were eroded by cavitation. The AM method selective laser melting (SLM) was used to manufacture at lower cost, but had seemingly lower erosion resistance. The cavitation erosion properties of Inconel 625 and 718 are studied as a function of hardness and surface roughness with the ASTM G134 cavitating jet. The samples were studied 3 surface conditions, as deposited/as used in applications, machined and polished, for both forged and AM manufacturing. Indentation reveals slight surface hardening for machined samples. X-ray diffraction (XRD) shows similar polycrystalline $\gamma$-Ni-based microstructure for all samples. Scanning electron microscopy (SEM) images of the cut cross-sections reveal the fractures and pits, as well as some porosity in the case of SLM samples. Images of the surfaces during erosion reveal some fracture mechanisms: machined samples erosion start quickly on pits and scratches. The SLM718 samples were found to have good cavitation erosion resistance if machined, while the SLM625 samples have comparatively poorer resistance. As-deposited samples have the lowest resistance, and surprisingly machined samples are more resistant than polished.
\end{abstract}

Keywords Additive manufacturing - Cavitation erosion · Inconel · Nickel-based · Superalloy · 3D printing

G. Taillon $(\bowtie)$

Department of Applied Mechanics, Waseda University,

Shinjukuku, Tokyo 169-8555, Japan

e-mail: taillon.gabriel@aoni.waseda.jp

K. Miyagawa

Department of Applied Mechanics and Aerospace Engineering,

Waseda University, Shinjukuku, Tokyo 169-8555, Japan

e-mail: k-miyagawa@waseda.jp

\section{Introduction}

The aerospace industry plays a role of ever increasing importance in the modern, globally connected world. According to the Federal Aviation Administration, an average of more than 2.7 million passengers flew in and out of U.S. airports everyday in 2018 [1]. Forecasts also indicate more passenger traffic in the long term, after a slow recovery from the global COVID-19 pandemic [2]. The Federal Aviation Administration's Office of Commercial Transportation (FAA AST) notes that the number of global orbital launches has steadily increased since 2004 [3]. Satellites are put into orbit to increase global connectivity, enabling, for example, the global positioning system (GPS). There are signs that the rocket launch market is to grow in the coming years, and new launch vehicles are being developed to address a latent need to launch small satellites and satellite constellations.

Jet engines were first manufactured using stainless steels, but materials with better high-temperature properties were soon designed $[4,5]$. Such materials include so-called superalloys which are characterized by advantageous mechanical properties: high creep resistance, exceptional strength, toughness, resistance to corrosion and oxidation, even at high temperatures (up to $1050{ }^{\circ} \mathrm{C}$ [6]). Ni-based superalloys, Inconels and Nimonics, for example, are the material of choice for jet engines [7].

Aerospace components such as turbines blades often have complex geometries that increase manufacturing costs. To address this issue, a number of manufacturing methods that produce parts layer by layer at lower costs have been developed [8]. These additive manufacturing (AM) methods melt and solidify a feedstock material in powder or wire at a precise location $[9,10]$. 
Using the AM method selective laser melting, Inconel 718 and 625 were formed into pipes used to transport cryogenic fluids in a rocket engine. After a cycle of operation, the pipes were found to be eroded by cavitation, i.e., vapor bubbles in a liquid under low pressure [11, 12]. These bubbles can cause material erosion through the formation of microjets and shockwaves, after implosion under higher pressure. The repeated impacts on the surface deform the surface leaving pits and eventually erode the material [13]. The erosion is unacceptable to the rocket manufacturers, which wish to better understand the erosion mechanisms of the SLM superalloys.

In the literature, there are numerous papers on manufacturing methods and erosion of Inconels. Unfortunately, cavitation is only a single type of erosion and there are numerous manufacturing methods, so studies specifically about cavitation erosion of SLM Inconels are relatively rare. Some example of slightly related studies include cavitation erosion of Inconel 625 coatings manufactured by cold spray [14] and the fatigue performance of Inconel 625 weld claddings [15]. As for Inconel 718, studies on its high temperature erosion [16], dry erosion wear performance [17] and slurry erosion [18] can be found.

There are numerous papers that present interesting results about the effects of the manufacturing methods on microstructure and mechanical properties of the Inconels. SLM tends to increase the proportion of secondary phases and features that grow in correspondence to the laser movement [9, 19]. Heat treatment changes the $\gamma^{-N i}$ sizes and favors the growth of fine-dispersed ${ }^{\prime} \mathrm{Ni3Nb}$ and $\gamma^{\prime}$ $\mathrm{Ni3}(\mathrm{Al}, \mathrm{Ti})$ precipitates $[20,21]$. This greatly increased Inconel 718 strength at room temperature. Also, the presence of the $\delta$ phase decreases the mechanical properties of the Inconel 718, which can be resolved by heat treatment that increases erosion resistance [22, 23]. Electrochemical polishing is shown to decrease the surface hardness of Inconel 718 manufactured by SLM due to it relieving internal stresses and dissoluting the $\delta$ phase [24].

The fatigue properties of Inconel 625 produced by SLM show that porosity contributes negatively to fatigue resistance [25]. Inconel 625 was previously studied using a Standard Test Method for Cavitation Erosion Using Vibratory Apparatus (ASTM G32) vibratory cavitation erosion apparatus and liquid impingement [20]. It was found that Inconel 625 is more resistant than Inconel 600 to both erosion mechanisms due in part by its superior hardness, though in these case they were not manufactured by an AM method.

Cavitation erosion tests of AM Inconels are rare, and this particular paper focuses on an application in which asdeposited tubes were used. For this reason, erosion resistance is characterized depending on surface conditions: machined, polished and as-deposited. The standard ASTM
G134 cavitating jet erosion apparatus is used to compare the resistance of SLM and forged Inconel samples because of its aggressiveness. Scanning electron microscopy enables the visualization of erosion damage on the surface, and the samples were cut after the tests to visualize crack formation. SEM images are taken before periodically during the erosion test to enable the visualization of some amount of the superalloy microstructure and to analyze the material damage and removal mechanisms. XRD was used to perform an analysis of the sample microstructure, in combination with the previously mentioned surface SEM images. Micro-indentation with a Vickers indenter was used to measure the surface hardness of the sample crosssections to determine if the surfaces were hardened, and to correlate material hardness with erosion resistance.

\section{Methodology}

\section{Material Properties}

The two materials studied satisfy SAE International standards: AMS5663N (Inconel 718) and AMS5666J (Inconel 625). Both are nickel-chromium based superalloys known for high corrosion, fatigue, creep and oxidation resistance in high-temperature conditions [6]. Alloys from this family are regularly used in aerospace and many other high performance applications.

The standards define tolerances for the composition, heat treatment, microstructure and mechanical properties of the superalloys. The chemical composition of the Inconel 625 and 718 is presented in Table 1. Both alloys have roughly the same amount of $\mathrm{Ni}, \mathrm{Co}$ and $\mathrm{Nb}$, while the 718 has much more $\mathrm{Fe}$ and $\mathrm{Ti}$ and the 625 has much more Mo. These differences lead the Inconel 625 to have good oxidation resistance and corrosion resistance in wet environments, high strength and good weldability. On the other hand, Inconel 718 has high creep and stress-rupture resistance up to $704{ }^{\circ} \mathrm{C}$ and good oxidation resistance up to $982{ }^{\circ} \mathrm{C}$.

The main phase types of interest for superalloys are:

- The austenitic Ni/Al $\gamma$ phase,

- The $\mathrm{Ni3Al}{ }_{\gamma}^{\prime}$ precipitate,

- The ${ }^{\prime \prime} \mathrm{Ni3Nb}$,

- The ${ }_{\delta} \mathrm{Ni} 3 \mathrm{Nb}$,

- Carbides and borides

Various other deleterious phases exist, including the Laves hexagonal phase that forms in iron- and cobalt-based superalloys after extended high-temperature exposure. Standard superalloys are free of these phases. Refer to [7, 26] for additional information. 
Table 1 Atomic composition of standard Inconel 625 and Inconel 718

\begin{tabular}{|c|c|c|c|c|c|c|c|c|c|c|c|c|c|c|}
\hline & & $\mathrm{Ni}$ & $\mathrm{Cr}$ & Mo & $\mathrm{Fe}$ & $\mathrm{Nb}$ & $\mathrm{Co}$ & $\mathrm{Mn}$ & $\mathrm{Si}$ & $\mathrm{Ti}$ & $\mathrm{Al}$ & $\mathrm{C}$ & $\mathrm{P}$ & $\mathrm{S}$ \\
\hline Inconel 625 & $\operatorname{Max}$ & Rem. & 23.00 & 10.00 & 5.00 & 4.15 & 1.00 & 0.50 & 0.50 & 0.40 & 0.40 & 0.10 & 0.015 & 0.015 \\
\hline (AMS5666J) & Min & Rem. & 20.00 & 8.00 & & 3.15 & & & & & & & & \\
\hline Inconel 718 & $\operatorname{Max}$ & 55.00 & 21.00 & 3.30 & Rem. & 4.15 & 1.00 & 0.35 & 0.35 & 1.15 & 0.80 & 0.08 & 0.015 & 0.015 \\
\hline (AMS5666J) & Min & 50.00 & 17.00 & 2.80 & Rem. & 3.15 & & & & 0.65 & 0.20 & & & \\
\hline
\end{tabular}

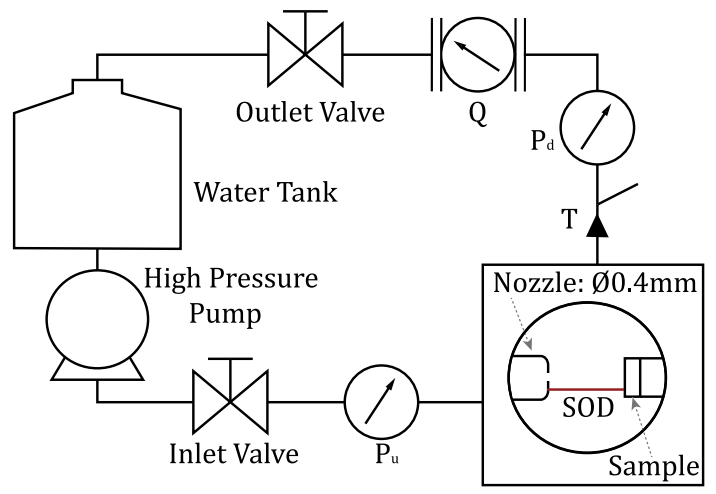

Fig. 1 Schematic of ASTM G134 compliant cavitation erosion jet

Table 2 Sample name, data marker and linestyle depending on manufacturing method, material and roughness

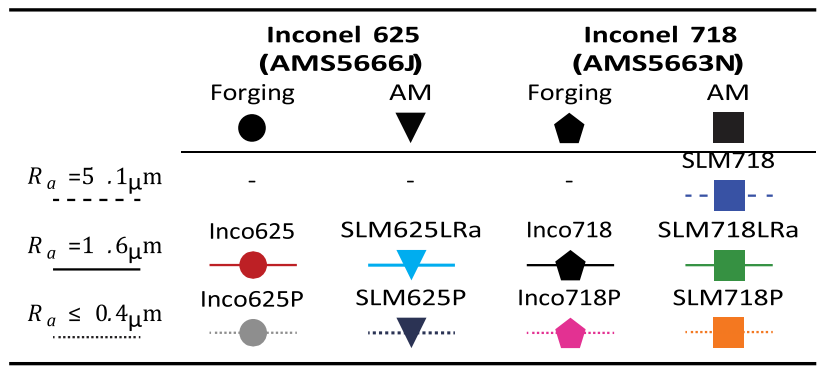

Samples with $R a<=0.4 \mu_{\mathrm{m}}$ were polished to a mirror finish.

The Inconel 625 is annealed, while Inconel 718 is solution and precipitation treated. The first superalloy's heat treatment is intended to completely recrystallize the material and increase ductility, weldability, homogenize the microstructure, etc. Solution treatment serves to dissolve the secondary phases which uniformizes the microstructure in preparation for the subsequent aging processes, which also increases corrosion resistance and offers maximum ductility. The latter precipitation treatment strengthens and relieves stress in the material by encouraging the formation of beneficial phases $\left(\gamma^{\prime}\right.$ and $\left.{ }_{\gamma}^{\prime \prime}\right)$ from the supersaturated $\gamma$ matrix obtained from the previous solution treatment.

The peaks for the $\gamma, \gamma$ and $\gamma^{\prime \prime}$ phases are known to overlap, but XRD measurements were performed in the

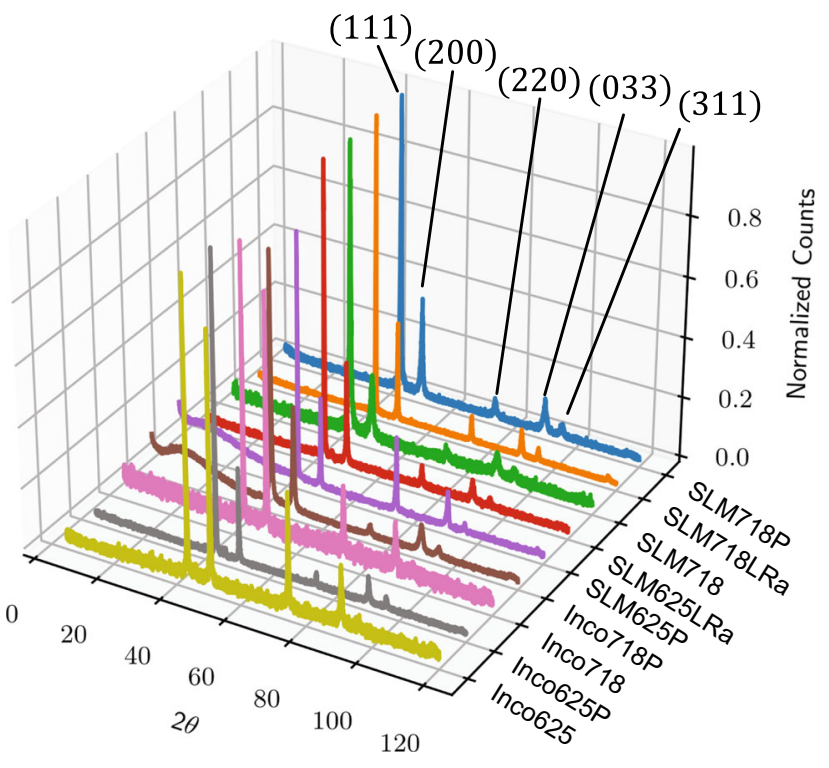

Fig. 2 XRD spectra for all samples are very similar

hopes of identifying other deleterious phases present in the AM samples.

Unfortunately, 3D printing may lead to material performance decrease compared to conventional machining through many different mechanisms. Chemical elements present in alloys may vaporize at different rates during melting, causing composition change, which directly affects material microstructure. Cracks can be introduced in the material at grain boundaries due to solidification cracking, similarly to welding. Porosity, unmelted 'voids' and cracking $[27,28]$ in the manufactured pieces constitute macro-scale defects that have an adverse effect on mechanical properties. In short, AM samples are expected to have diminished cavitation erosion resistance.

\section{Cavitation Erosion Jet}

The ASTM G134 Standard Test Method for Erosion of Solid Materials by Cavitating Liquid Jet (ASTM G134) is used to perform erosion tests. The cavitation bubbles are carried by the flow to the sample surface, imploding along the way. The cavitation jet is known to be aggressive, so it 
was chosen to perform erosion tests on the particularly strong and tough superalloys.

The schematic of the present cavitation erosion jet is shown in Fig. 1. A number of sensors, including upstream and downstream pressure sensors and flow sensors, are used to monitor the flow. The jet's nozzle must have a precise geometry with a diameter of $0.4 \mathrm{~mm}$. The water temperature is maintained in the interval $35 \pm 1{ }^{\circ} \mathrm{C}$. The upstream pressure is maintained at $20 \mathrm{MPa}$, while the downstream pressure is modified to control the cavitation number $\sigma$ :

$\sigma=\frac{p_{d}-p_{v}}{\frac{\rho V^{2}}{2}}$

with $\rho$ the fluid density, $p u$ the upstream pressure, $p d \sigma=$ $\frac{p_{d}}{p_{u}}$ the downstream pressure and $v$ the fluid velocity, which can be simplified to. Experiments are performed at conditions that maximize the erosion rate previously determined to be a stand-off distance (SOD) of $11 \mathrm{~mm}$ and $\sigma=0.02$.

Mass loss was measured every 10min until $2 \mathrm{~h}$ of erosion time, then every $30 \mathrm{~min}$ until a final erosion time of ${ }_{5 \mathrm{~h}}$ is achieved. Following each erosion step, the samples were cleaned in an ultrasonic bath using acetone, dried, then weighed using a scale with a $0.01 \mathrm{mg}$ precision. A total of 3 repetitions were performed for each sample. SEM pictures were taken every $20 \mathrm{~min}$ interval, until ${ }_{2 \mathrm{~h}}$ of total erosion time, well after the incubation period is over.

\section{Polishing}

To transport cryogenic fluids, Inconel 718 was deposited by SLM forming pipes. The laser powder bed fusion (LPBF) method, otherwise known as selective laser melting (SLM), produces samples that have a rather high roughness, $R a=5.1 \mu \mathrm{m}$. Forged samples and AM samples were also manufactured to a $R a=1.6 \mu \mathrm{m}$ flat finish by machining, noted as LRa samples. Then, the ASTM G134 also suggests polishing samples for the erosion tests. In total, 3 different roughness are analyzed, polished $R a<0.4 \mu_{\mathrm{m}}$, machined $R_{a}=1.6 \mu \mathrm{m}$ and $\mathrm{AM}$ as deposited $R a=5.1 \mu_{\mathrm{m}}$. Cavitation erosion is performed for 3 different surfaces and two manufacturing methods, as shown in Table 2. This table also presents the data marker shapes and sizes, as well as curve colors used in graphs throughout this article.

The polishing methodology to achieve a mirror finish goes as follows. Samples are first polished using $\mathrm{SiC}$ mats, followed by a fine polish using diamond particles in solution. The $\mathrm{SiC}$ mats used have a grit up to 800 , followed by two sizes of diamond suspensions: $9 \mu \mathrm{m}$ and $3 \mu \mathrm{m}$. The polished samples have a scratchless mirror finish, with a roughness equal or lower than the standard $0_{.4} \mu_{\mathrm{m}}$ requirement.

Some samples were cut to perform hardness measurements on sample cross-sections. Diamond-wire saw cutting was too slow to cut samples within a reasonable timeframe,

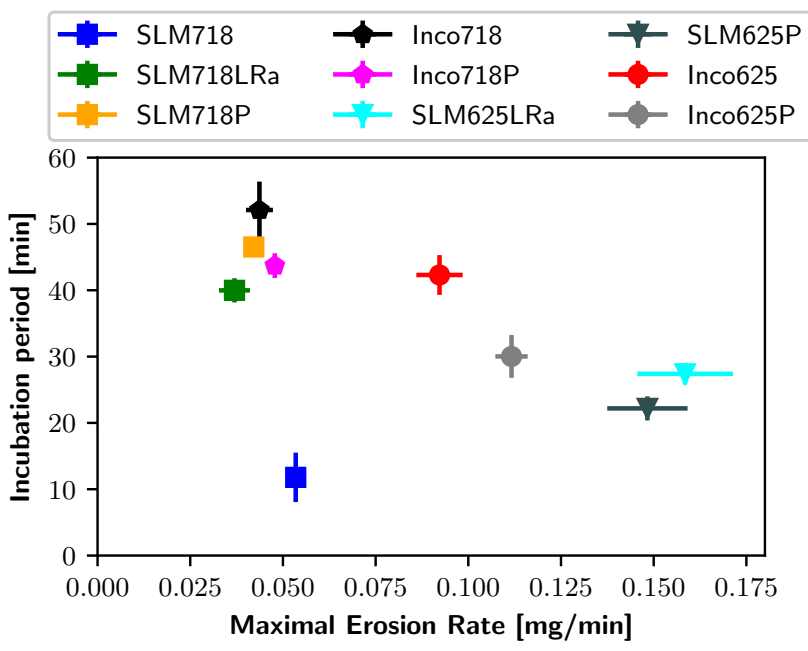

Fig. 4 Superalloys' with high erosion rate tend to have lengthier incubation periods. Minimal error to show error bars: $0.0025 \mathrm{mg} / \mathrm{min}$ in the $\mathrm{X}$ direction and $1.75 \mathrm{~min}$ in the $\mathrm{Y}$ direction
Fig. 3 Mass loss for all samples. Absent error bars imply an error of less than $\pm 0.75 \mathrm{mg}$

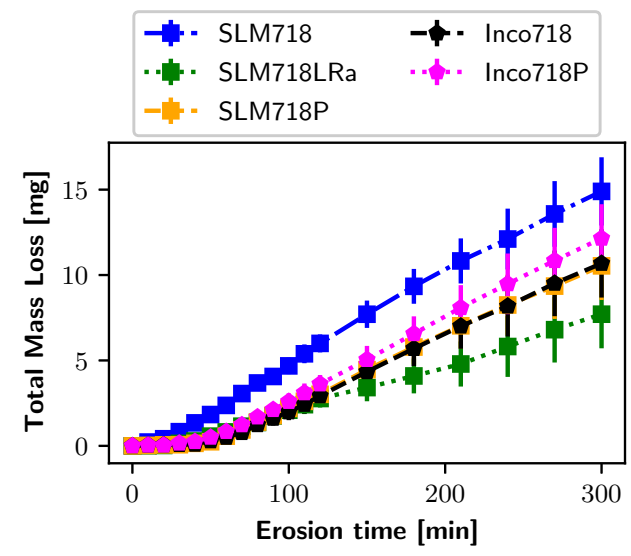

(a) 718 samples

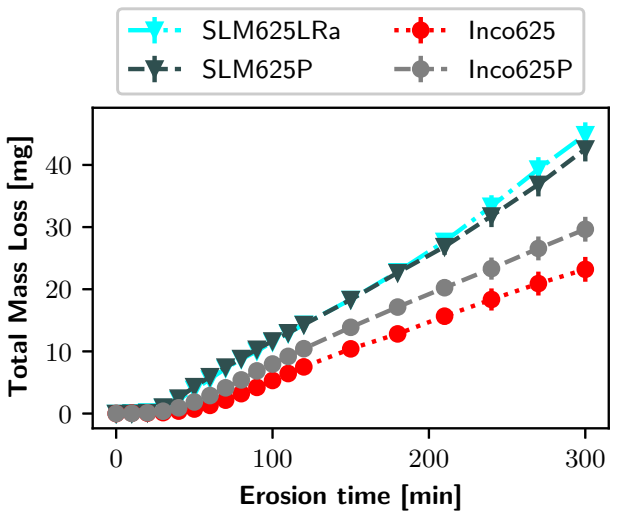

(b) 625 samples 


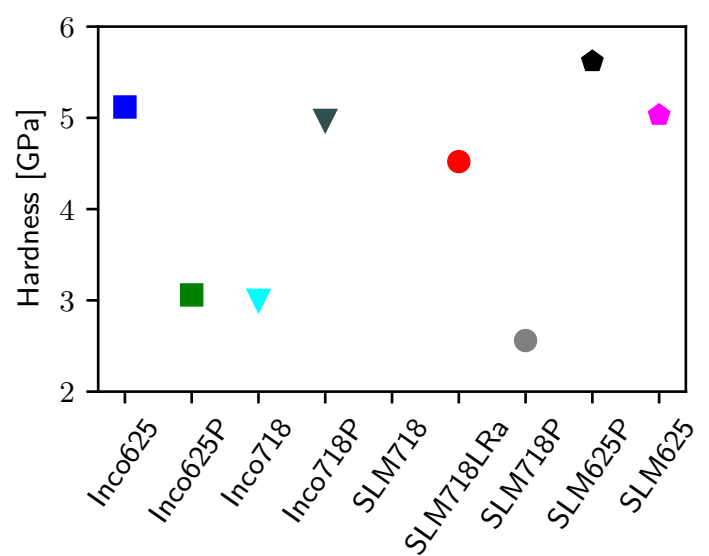

Fig. 5 Surface hardness of all samples. As-deposited SLM718 is too rough to be indented

so an industrial cutter was used instead. The round crosssections were then encased in bakelite for easier manipulation during subsequent polishing, imaging and indentation testing.

\section{Indentation Measurements}

Samples were measured using a Vickers tipped microindenter, following the ASTM E384 standard. The surface hardness is determined by measuring the average diagonal length of the imprint left by the indenter. Both the surfaces and polished cross-sections of the samples were measured, except for the surface of the SLM sample which was too rough to be measured with the micro-sized indenter.

To determine if the sample's surface was hardened, a number of indentations were performed on the cross-sections, up to $500 \mu \mathrm{m}$ away from the surface of interest, starting from a distance of $50 \mu \mathrm{m}$ from the surface. Indentations were performed to always keep the distance between samples be always greater than two and a half times the Vickers diagonal, as specified in the E384 standard. In the current case, the diagonals were approximately 30- $40 \mu_{\mathrm{m}}$ in length, so the indentations were also shifted in the perpendicular axis.
Fig. 6 Cross-section hardness for all materials as a function of indentation depth

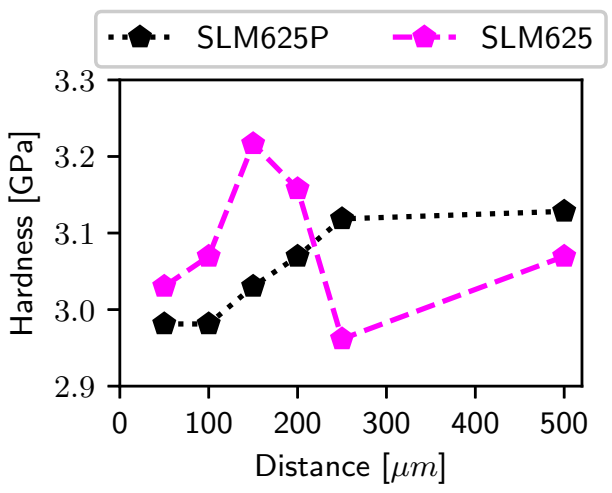

(a) SLM625

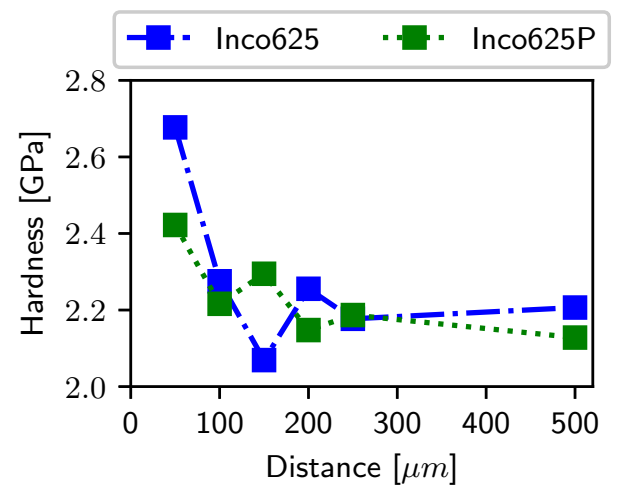

(c) Inco625

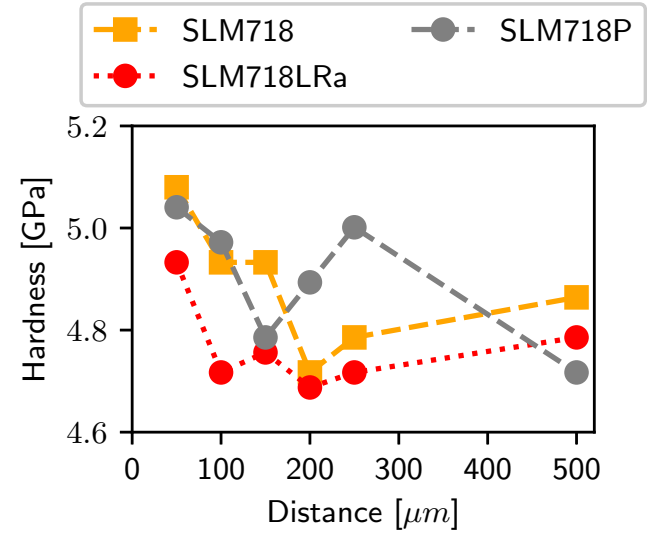

(b) SLM718

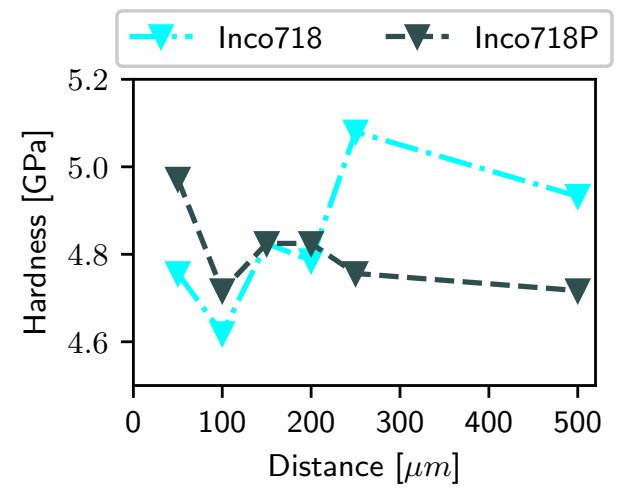

(d) Inco718 


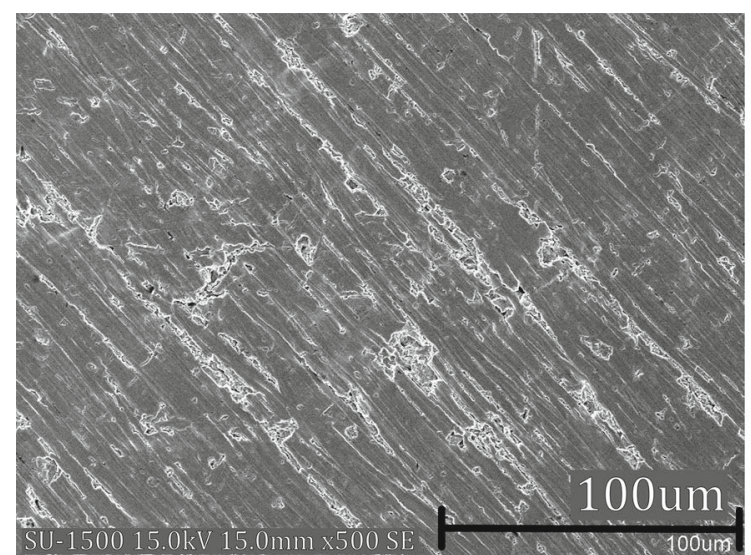

(a) $20 \min , 500 \times$

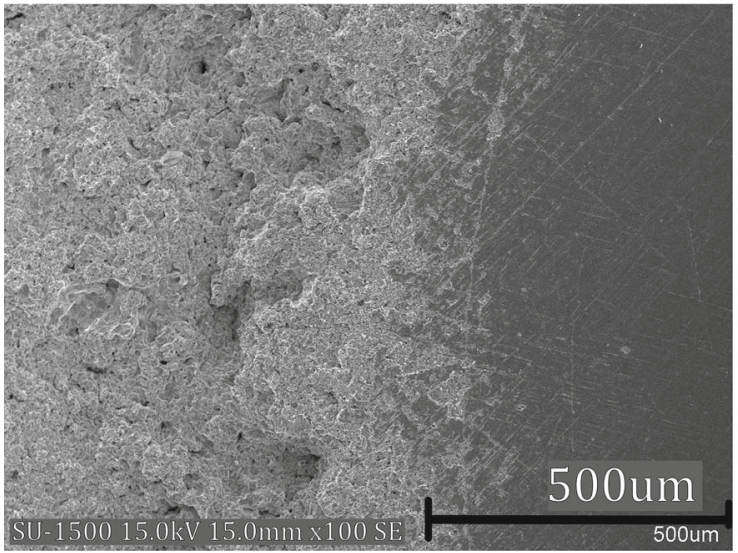

(c) $80 \min , 100 \times$

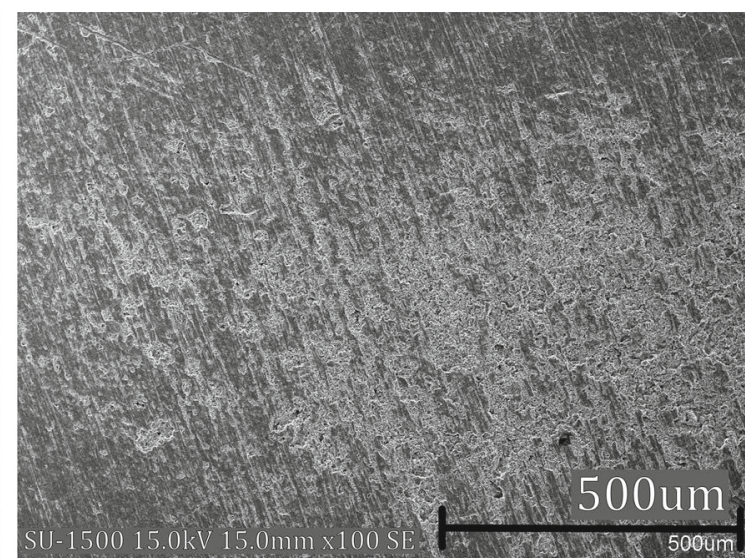

(b) $40 \mathrm{~min}, 100 \times$

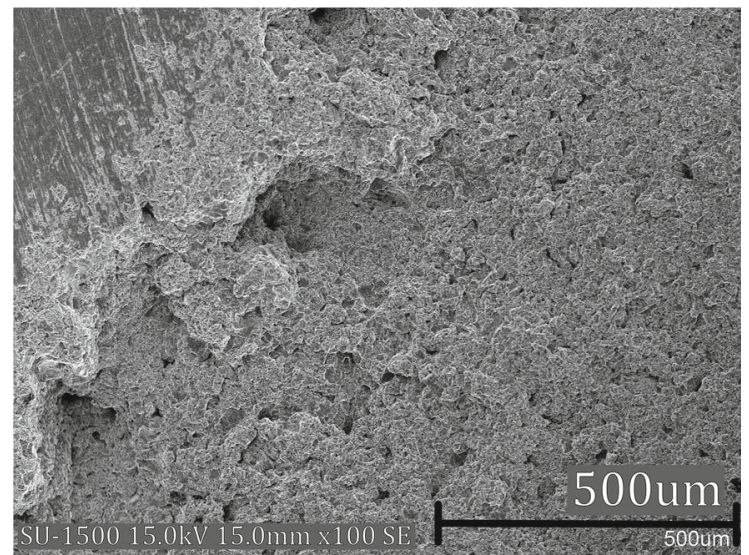

(d) $120 \mathrm{~min}, 100 \times$

Fig. 7 SEM pictures of surface of Inconel 625 as a function of erosion time.

\section{Results and Discussion}

Firstly, the XRD spectra of the materials are presented, followed by the cavitation erosion results. Then, the SEM surface images and indentation measurements will be used to analyze the erosion mechanisms.

\section{XRD}

The XRD spectra are shown in Fig. 2. The same series of peaks mostly associated with the $\gamma$-Ni phase is visible for all materials. The peaks associated with the $\gamma^{\prime}$ and $\gamma^{\prime \prime}$ phases are not differentiable from the overpowering $\gamma$ peaks, as they are known to overlap for a large range of $2 \theta$ degrees [29, 30]. According to [29], $\gamma^{\prime}$ and $\gamma^{\prime \prime}$ grains in commercial Inconel 718 samples manufactured by SLM are too small to be resolved even by high resolution SEM.

In some of the spectra, a small double-peak between the two major peaks at $43^{\circ}$ and $51^{\circ}$ can be observed. The phases that can be reasonably associated with these peaks are only impurities: $\mathrm{Fe} 2 \mathrm{Si}$, AlSi, CrTi, or others [31].

\section{Cavitation Erosion Tests}

Erosion tests are repeated 3 times, leading to the averaged total mass loss curves shown in Fig. 3. The measured incubation periods and maximal erosion rates computed using these curves are shown in Fig. 4. The cavitation erosion results mostly go as expected: higher roughness leads to less resistance and SLM samples are generally less resistant. Roughness is associated with more erosion because surface defects tend to serve as sites for accelerated cavitation damage [32] and introduce surface irregularities which create high stress regions that promote fatigue cracks [33, 34].

As expected, the as-deposited SLM718 sample is less resistant than its lower roughness SLM718LRa and SLM718P counterparts. Otherwise, the SLM samples manufactured from Inconel 718 have surprisingly good 


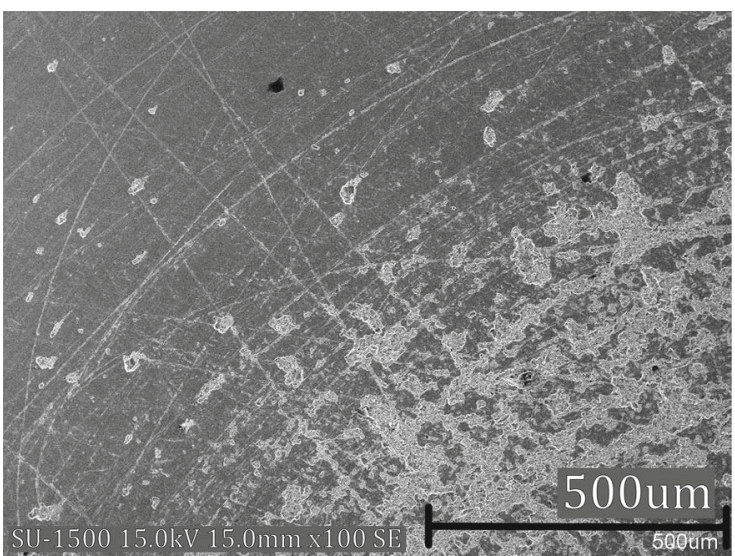

(a) $40 \min , 100 \times$

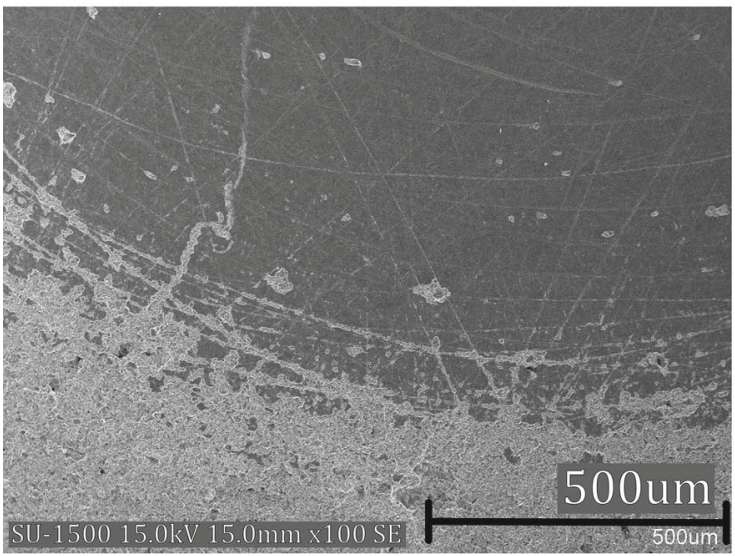

(c) $80 \mathrm{~min}, 100 \times$

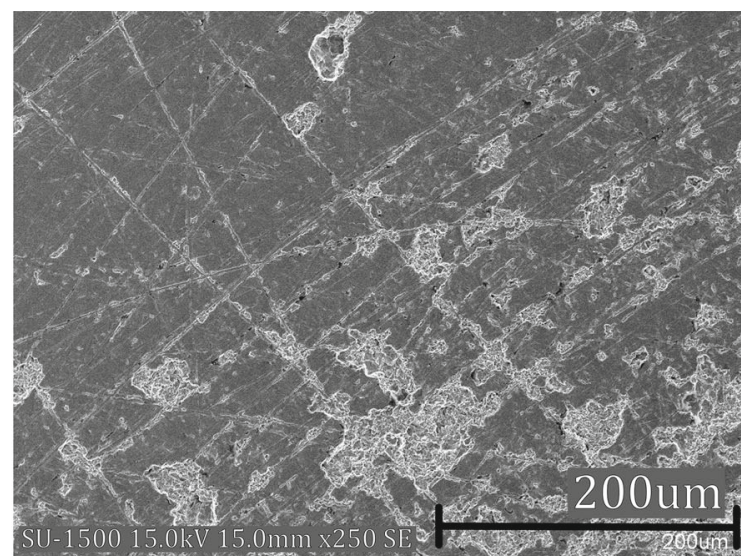

(b) $40 \mathrm{~min}, 250 \times$

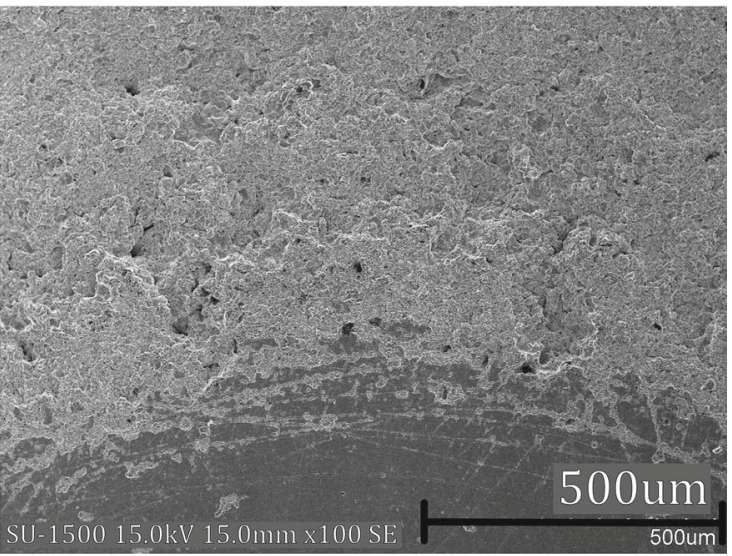

(d) $100 \min , 100 \times$

Fig. 8 SEM pictures of surface of Inconel 718 as a function of erosion time

cavitation erosion resistance. The machined SLM718LRa has a comparatively low erosion rate, very slightly lower than both forged Inco718 samples. Surprisingly, the average erosion rate of the polished Inco718P is lower than the machined Inco718, though both datasets are included in each other's errors. The errors for the 718 samples are generally high, so the $-1 \sigma$ of the erosion rate of the SLM718LRa is still higher than the $+1 \sigma$ rate of the Inco718 as shown in Fig. 4.

Inconel 625 results demonstrate much less variance and significantly lower resistance. The AM 625 samples have shorter incubation periods and higher erosion rates compared to Inconel 718. Both forged samples' cavitation erosion resistance actually decreased when polished (Inco718P and Inco625P), compared to their machined counterparts (Inco718 and Inco625). One possible cause of better cavitation erosion resistance for machined samples might be surface hardening, which is known to occur during conventional machining of superalloys [26]. The influence of surface hardening wanes as erosion time increases [11]. Anyhow, hardened surfaces can be removed by polishing, which would explain the higher erosion resistance of the machined samples. Microindentation hardness tests on sample cross-section and polished surfaces were performed to test this hypothesis in the following section.

For proponents of AM, these results are quite good news: simple surface machining to reduce roughness increases the cavitation erosion resistance of 3D printed parts significantly, in some cases up to conventionally manufactured and machined parts' performance. In the case of applications to rocket engines specifically, parts may be used only once for a very short time, which increase the importance of the incubation period. Machining of AM parts can more than quadruple the incubation period. Also, it seems that polishing is slightly detrimental to the cavitation erosion properties of Ni-based superalloys compared to conventional cutting and machining. Although any surface preparation appears significantly better than none. 


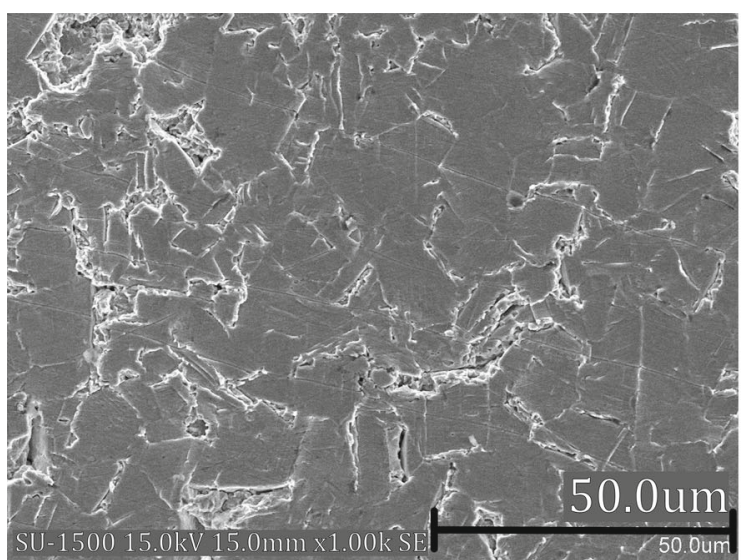

(a) $20 \min , 1000 \times$

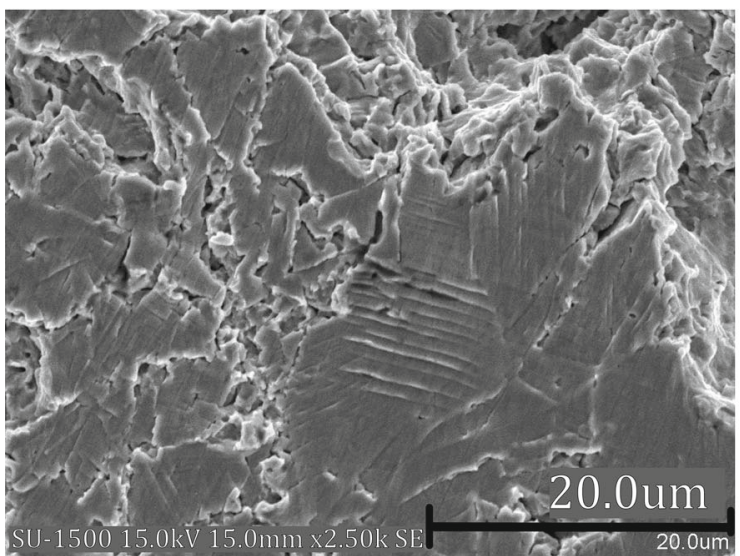

(c) $40 \min , 2500 \times$

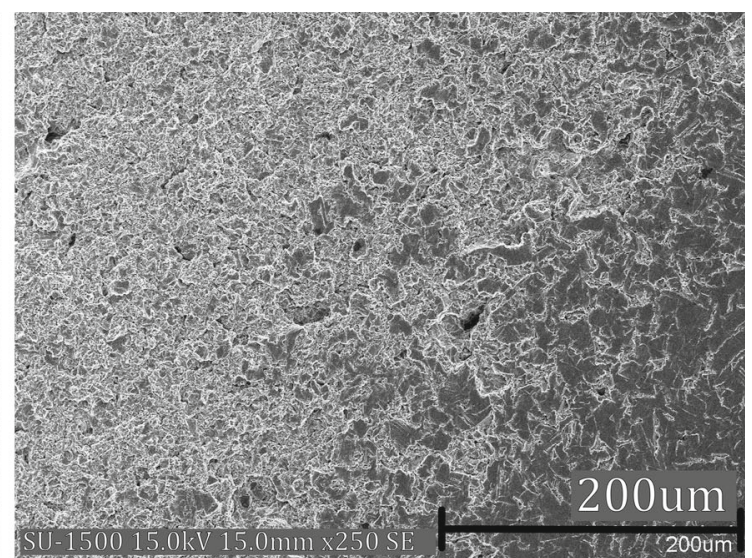

(b) $40 \mathrm{~min}, 250 \times$

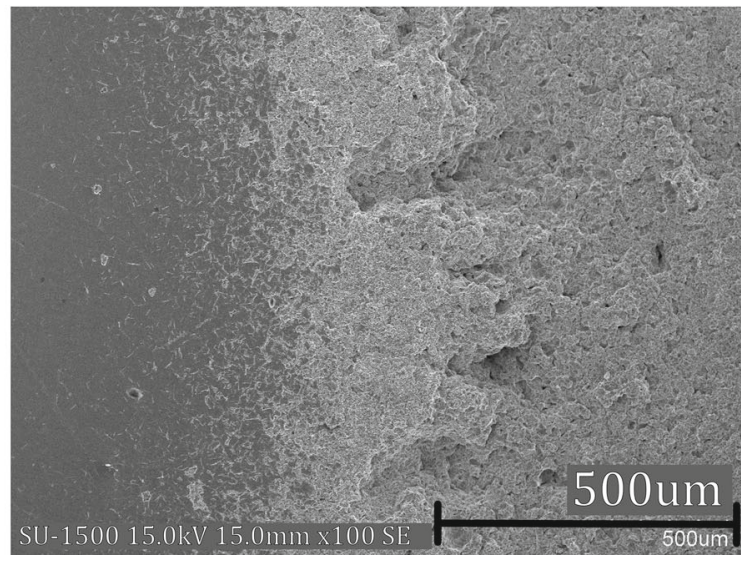

(d) $120 \min , 100 \times$

Fig. 9 SEM pictures of surface of polished Inconel 718 as a function of erosion time

\section{Indentation Results}

The expected hardness according to the SAE International standards are: AMS5663N (Inconel 718) and AMS5666J (Inconel 625) standards is no lower than 2.72GPa for Inconel 625 and no lower than 3.18GPa for the Inconel 718.

The surface indentation results are presented in Fig. 5 and for the material cross-sections in Fig. 6. The surface hardness of the polished Inconel 625 and SLM samples is lower than expected, others are consistently and significantly higher.

Also observable in Fig. 6, both unpolished Inconels' surfaces seem to be hardened, especially in the case of the Inconel 625. Inco625P is also slightly hardened. All Inconel samples that have similar hardness tend to have similar erosion rates, except the as-deposited sample which is exceptionally rough and vulnerable to cavitation erosion.

Surface hardening is most prominent in the case of the Inconel 625, where both the surface hardness and erosion resistance significantly decreased after polishing. The surface hardness of the polished Inconel 718 is also slightly lower, with an associated higher erosion rate. For longer erosion times, the performance of the unpolished Inconel 718 and 625 is expected to decrease to similar levels, when the surface material is completely removed. In general, rocket engines are used a single time and not for an extended time period. Briefly, polishing surfaces are very slightly less hard than their conventionally machined counterparts.

\section{Erosion Mechanisms}

SEM pictures of the eroded surfaces are taken every 20min during the first 2 hours of erosion. Samples are cut after the tests, and pictures of the polished cross-sections are presented.

Starting off with forged samples surfaces, Fig. 7 shows damage on the machined Inco625 samples accumulating over the erosion time. The low erosion time pictures show that the damage follows the surface machining pattern and occurs preferentially in visible scratches. Material removal 


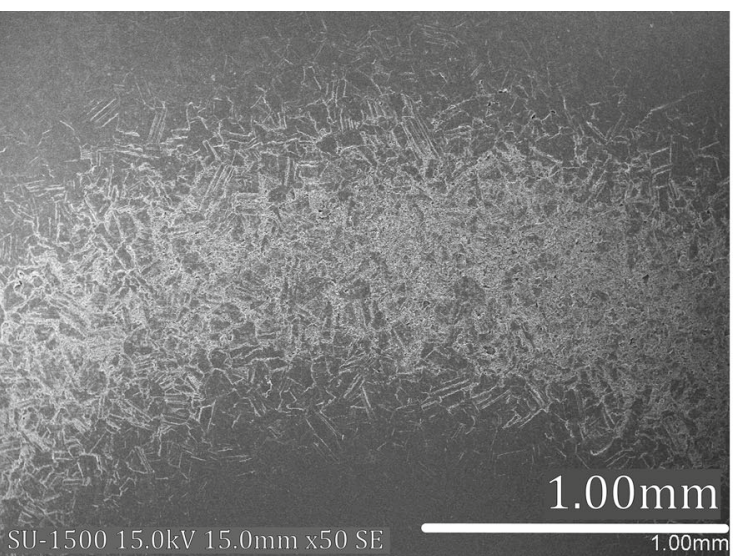

(a) $20 \mathrm{~min}, 50 \times$

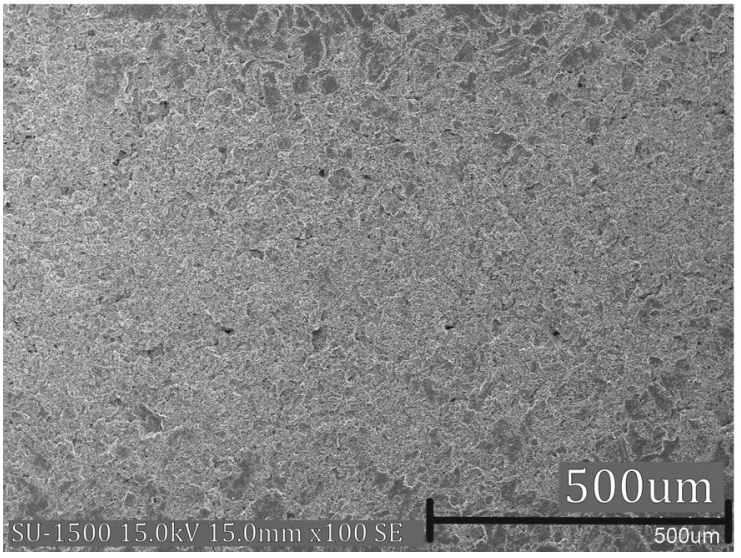

(c) $60 \min , 100 \times$

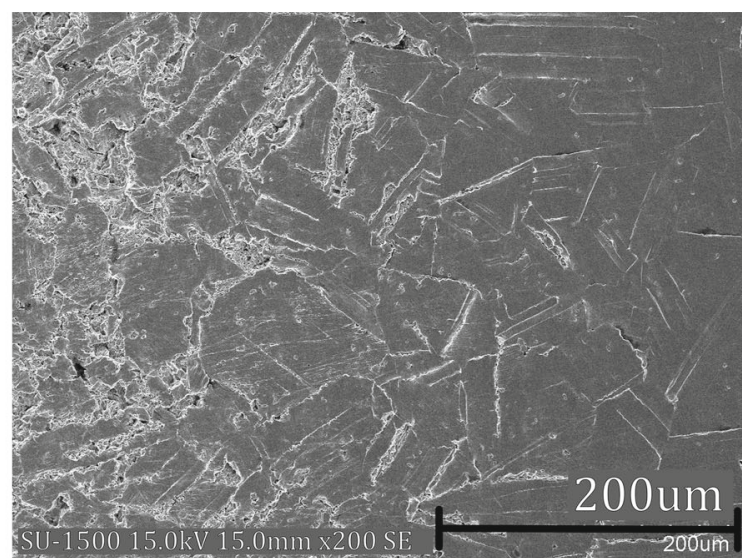

(b) $20 \min , 250 \times$

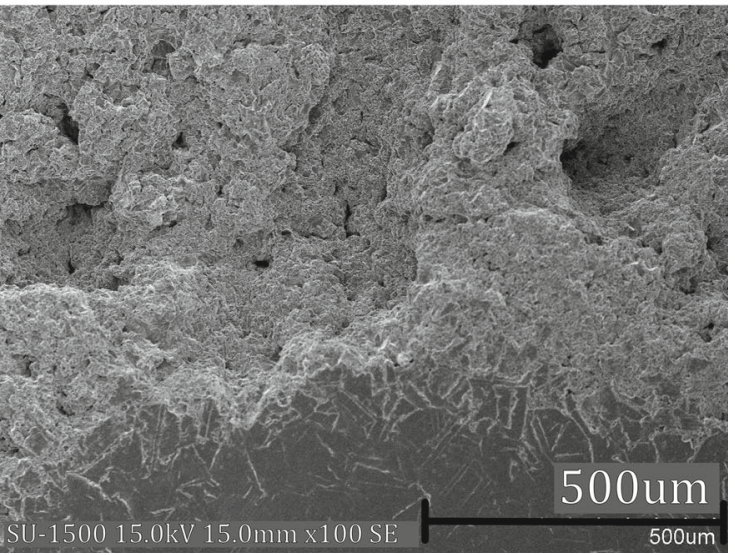

(d) $100 \mathrm{~min}, 100 \times$

Fig. 10 SEM pictures of surface of polished Inco625P as a function of erosion time

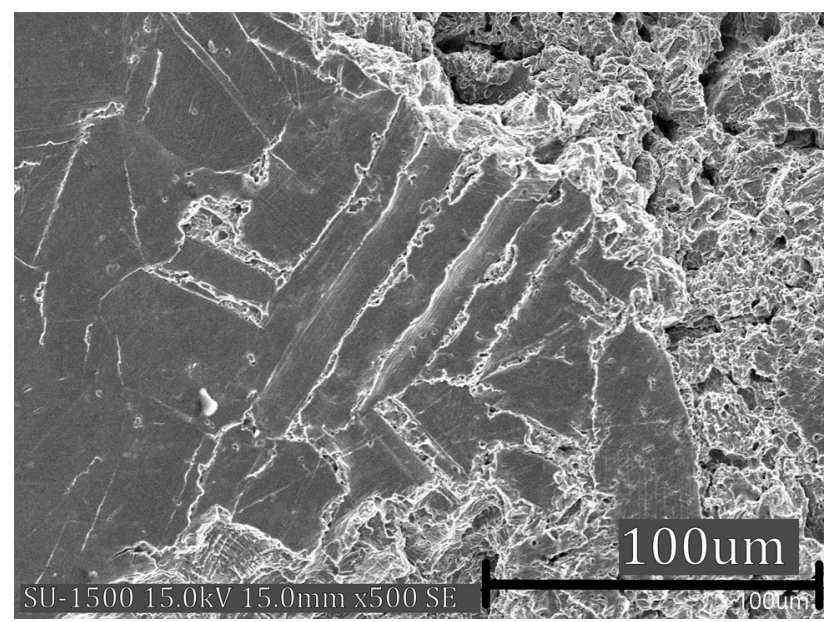

Fig. 11 Visible cleavage striations on a polished Inconel 625 samples after $100 \mathrm{~min}$ erosion $(\times 500)$

starts quickly, being observable after the first 20min, but these small holes' contribution to the measured mass loss is negligible. The same is observed for the machined Inco 718 samples in Fig. 8.
The polished samples contrast directly with this observation, as shown in Figs. 9 and 10. For both Inco718P and Inco625P, the initial damage accumulation phase seems to last longer without mass loss, with pits and cracks being generated at what appears to be grain boundaries. Some parallel lines appear on the surface, which demonstrates that cavitation erosion may cause cleavage of the crystallographic planes. These are sometimes referred to as cleavage striations [35].

For nickel-based superalloys, fatigue is known to be essentially a cleavage process [36], which fits with the nature of the cavitation erosion process. The repeated impacts cause irreversible dislocation movement, promote dislocation nucleation and eventually crack formation between crystallographic planes such as in Fig. 10 and in Fig. 11, or at grain boundaries and other weaknesses.

Figure 11 shows a zoomed-in picture of a set of cleavage striations. Cleavage planes are known to be areas of relative weakness in the materials which can promote the propagation of cracks and cause brittle fracture $[35,37,38]$. This fracture mechanism is generally more 

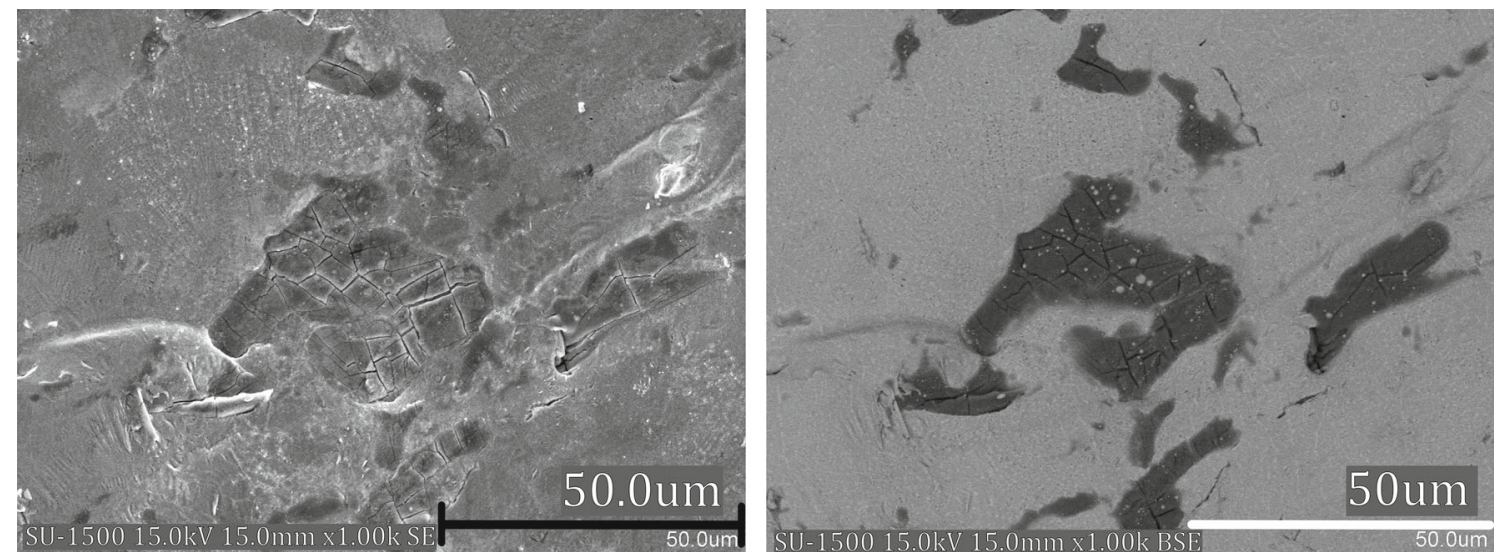

(a) $\mathrm{SE}, 0 \mathrm{~min}, 1000 \times$

(b) BSE, 0 min, $1000 \times$
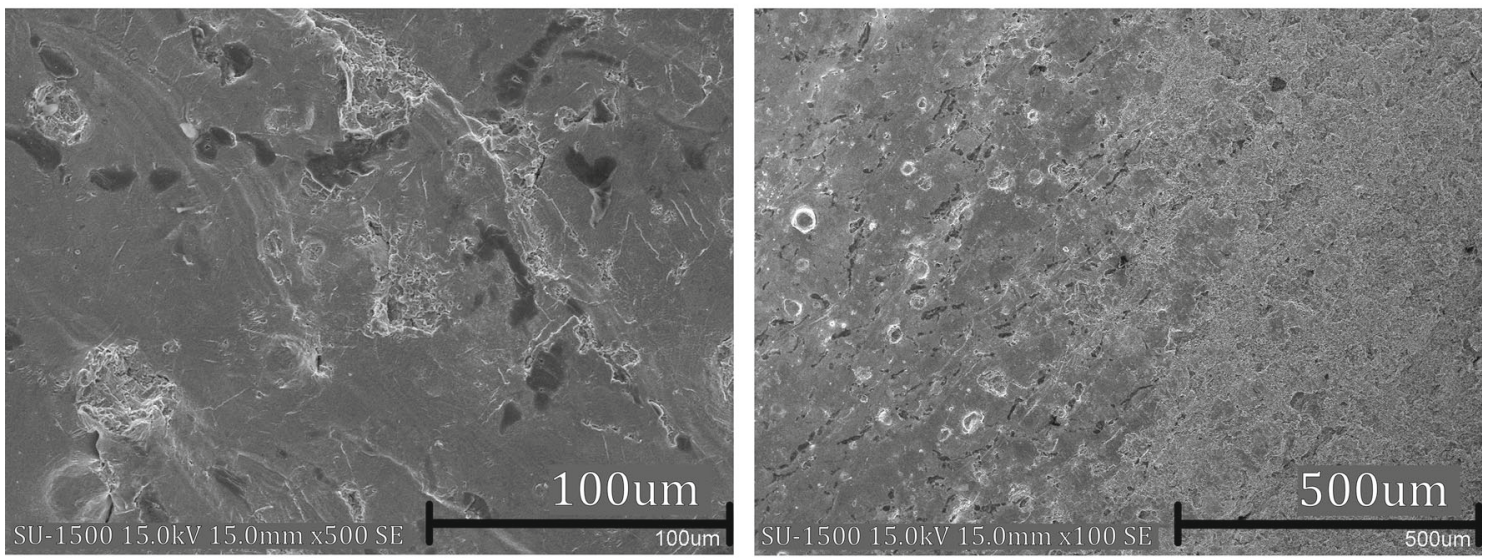

(c) $20 \min , 500 \times$

(d) $40 \mathrm{~min}, 100 \times$
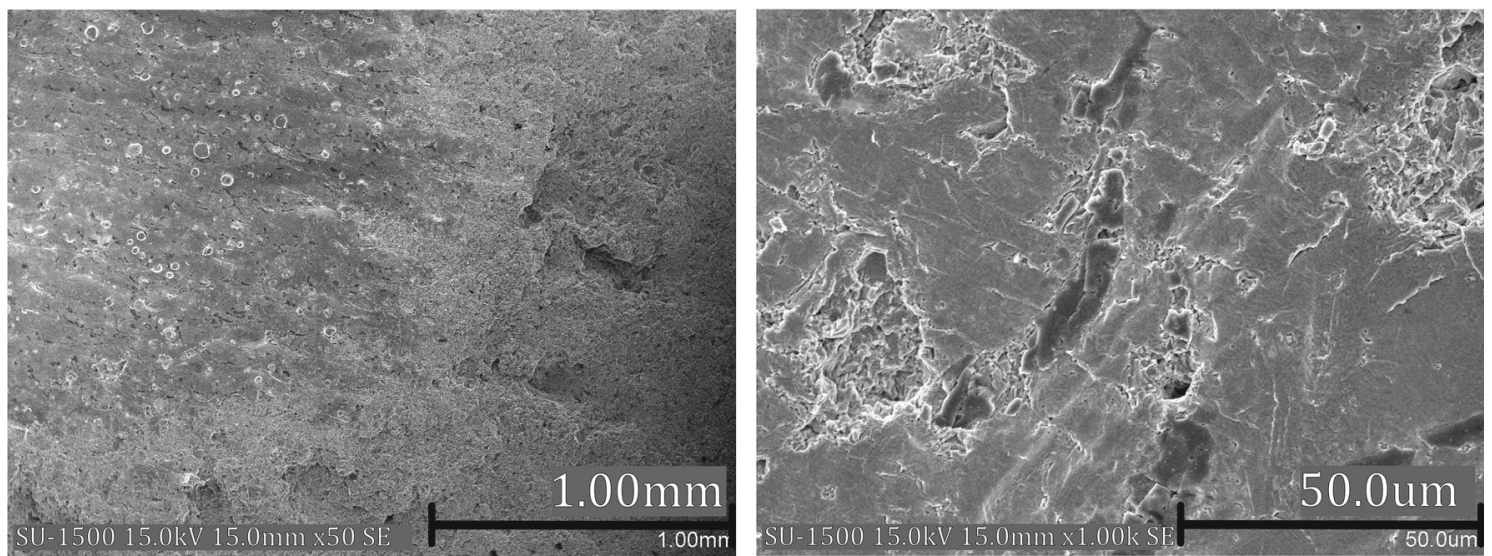

(e) $80 \min , 50 \times$

(f) $100 \min , 1000 \times$

Fig. 12 SEM pictures of surface of SLM718 as a function of erosion time 


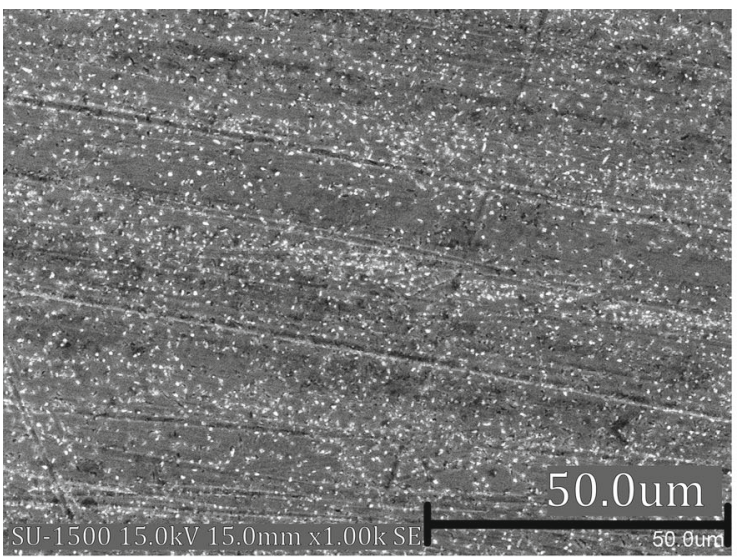

(a) $0 \min , 1000 \times$

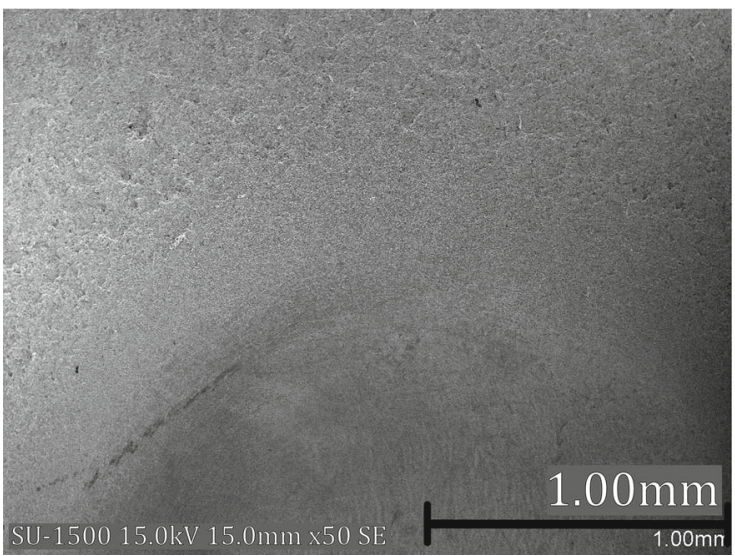

(c) $100 \min , 50 \times$

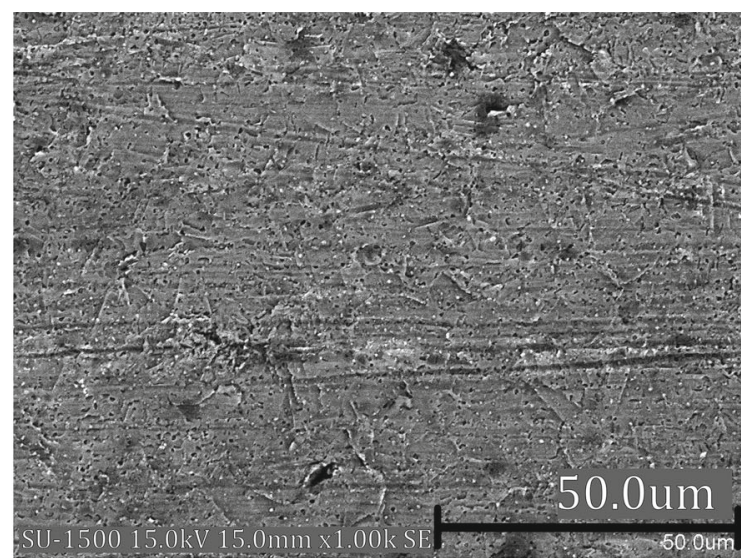

(b) $40 \min , 1000 \times$

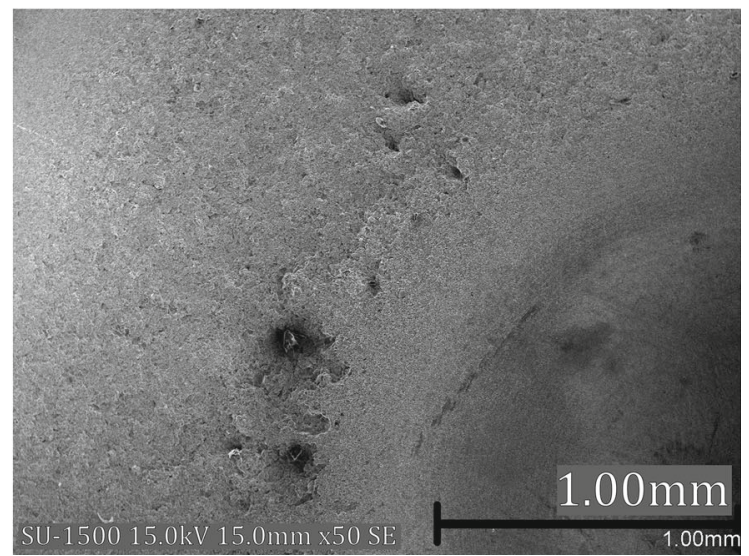

(d) $120 \mathrm{~min}, 50 \times$

Fig. 13 SEM pictures of surface of SLM718LRa as a function of erosion time

important for monocrystals, in which there are no grain boundaries. This is experimentally confirmed due to their relative scarceness compared to grain boundary cracks and small pits. The same cleavage striations can be also observed in the polished Inconel 718 samples surface SEM pictures in Fig. 9(c) for example, but are hardly observed on the surface of SLM samples.

Speaking of which, the SLM718 pictures reveal black grains embedded in a gray metal matrix in Fig. 12. Such grains are much more rare for SLM625 samples, see Fig. 16 for instance. Anyhow, for SLM718 samples, these grains themselves are often cracked, with smaller white grains and voids visible. All these surface defects lead to the much lower incubation period (half or less), and erosion rate of the SLM samples compared to all forged Inconel 718 samples and other low roughness SLM718LRa and SLM718P samples.

SLM samples have generally lower incubation period, which is reflected in surface pictures with removed material at shorter erosion times. At higher erosion times, the erosion pattern follows the typical, fractal erosion holes observable for cavitation erosion, as shown in 12(e). Focusing on the edge of the eroded region, such as in Fig. 12(f), reveals many erosion mechanisms: deep holes, parallel fissures which may be cleavage striations, fissures around and in the black grains...

As for the nature of the observed black and white grains, $\gamma^{\prime}$ and $\gamma^{\prime \prime}$ are strengthening phase of Inconel 718 is [39], although SEM pictures alone are insufficient to properly identify the observed phases. Still, the authors infer that the metal darker grains are probably $\gamma^{\prime}$ and the white grains the $\delta$ phase. This latter orthorhombic phase is known to precipitate either in the grain interior [31], or at grain boundaries [40]. As presented in Sect. 3.1, the XRD peaks visible at $43^{\circ}$ and $51^{\circ}$ in the measured data indicating the presence of deleterious phases in the SLM718, SLM718LRa and Inco718P samples.

To compare with the as-deposited SLM718, the polished SLM718P pictures in Fig. 14 and the machined SLM718LRa surface pictures shown in Fig. 13. Cracks are visible during the incubation of both samples. Many more surface defects are present on the surface of the 


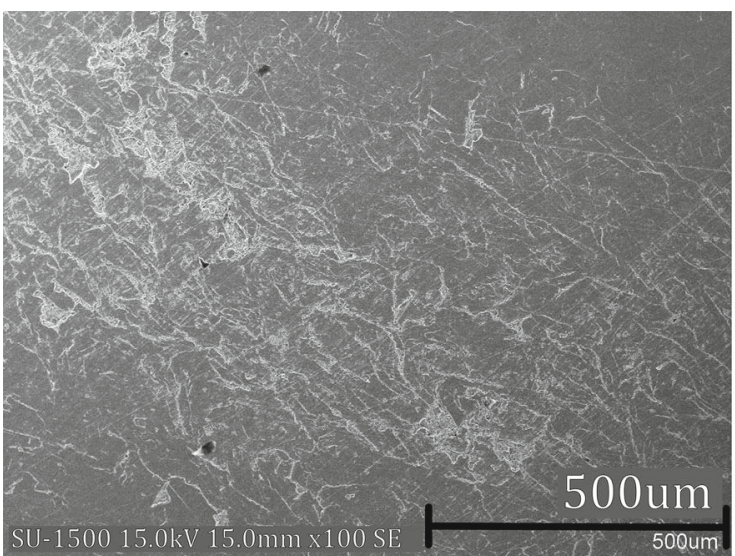

(a) $20 \min , 100 \times$

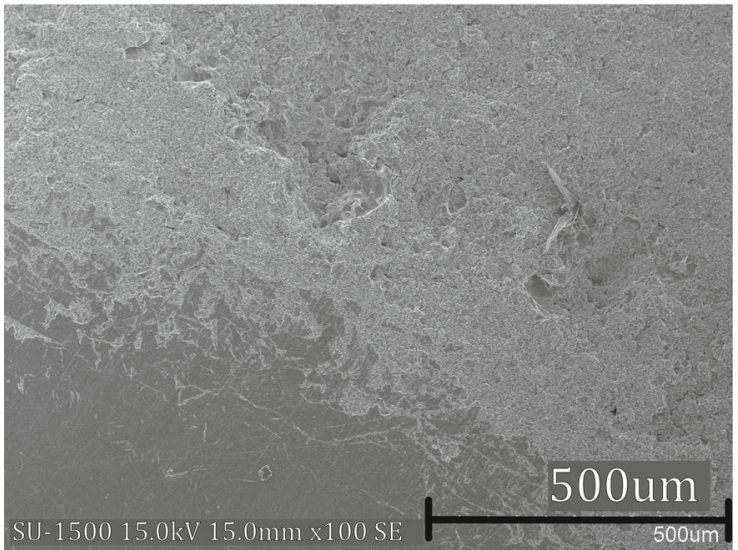

(c) $80 \min , 100 \times$

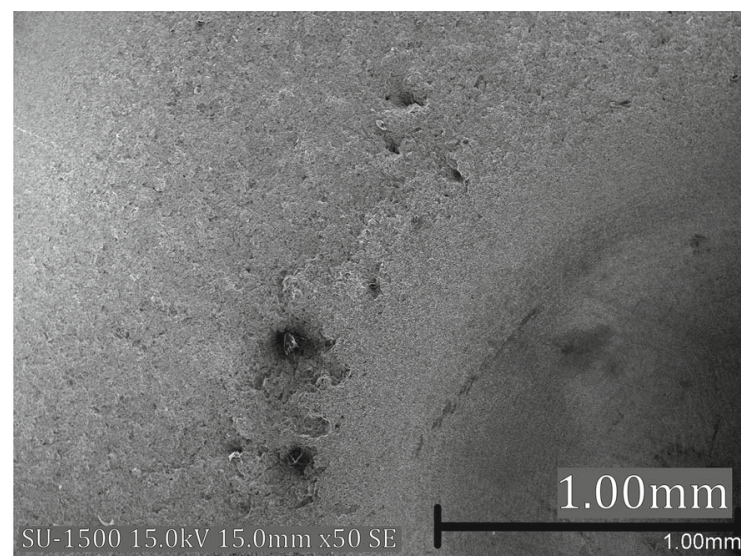

(b) $60 \mathrm{~min}, 50 \times$

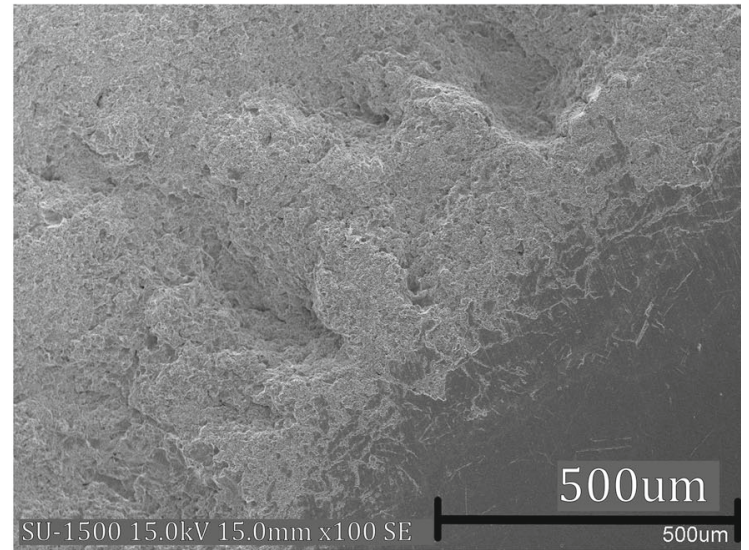

(d) $120 \mathrm{~min}, 100 \times$

Fig. 14 SEM pictures of surface of SLM718P as a function of erosion time

SLM718LRa due to the presence of the small white grains. After an erosion time of 20min, as shown on Fig. 13(b), these white grains were removed leaving black holes approximately ${ }_{10} \mu_{\mathrm{m}}$ in diameter. At $20 \mathrm{~min}$, bigger mass loss holes such as for the SLM718 sample are not yet observed. The white grains get eroded preferentially, while damage is still accumulating in the metal matrix.

Small white grains visible on the SLM718LRa and SLM718 samples are not visible on the SLM718P sample, as shown in Figs. 13(a) and 14(a). Only a small few black grains are visible on the polished surface. The absence of white grains and the comparatively small amount of observable black grains on the SLM625 surfaces prevent these erosion mechanisms, which might decrease its resistance. As shown in Fig. 15, repeated impacts lead to fractures and fissures on the surface and eventually erosion. Both machined SLM show faster material removal on the visible surface scratches (Figs. 16, 17).

The presence of what appears to be many defects on the surface of the SLM718LRa belies its superior cavitation erosion resistance. Still, its microstructure indicates mechanisms that may help explain its superior properties. The average crack length on the surface of SLM718LRa samples is somewhat short compared to other samples manufactured by AM. Cracks are also more numerous than other samples, indicating that more energy is necessary to cause the removal of significant amount of mass. The white grains get removed very quickly during the erosion process, but seemingly contributed only negligible mass loss compared to the other phases.

Ultimately, the drop in performance of the SLM718P sample compared to the SLM718LRa is much less significant than the as-deposited SLM718. It seems that surface machining of AM superalloys samples brings back the cavitation erosion resistance up to the resistance level of conventionally manufactured materials. 


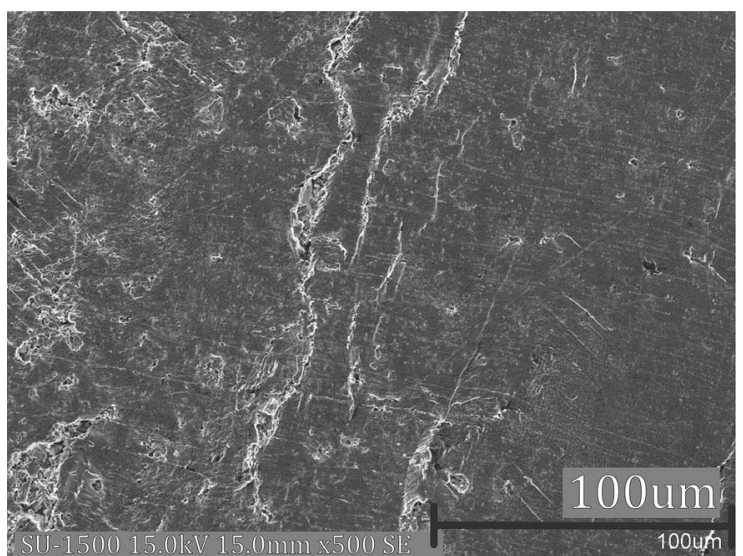

(a) $20 \min , 500 \times$

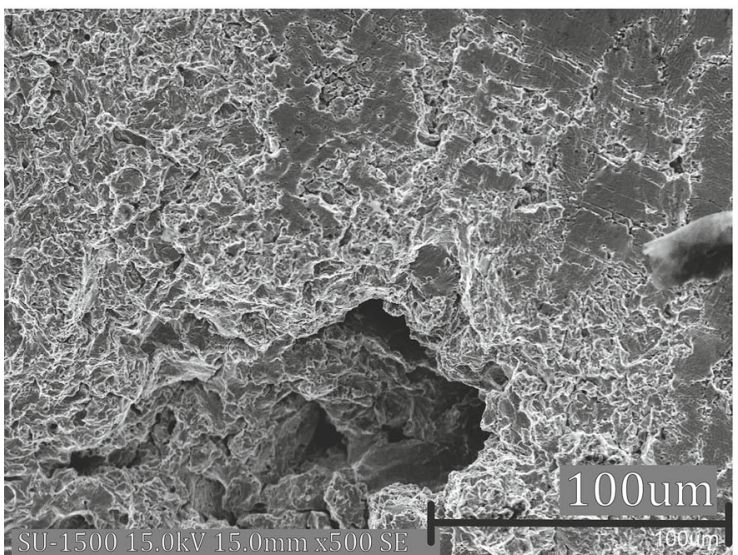

(c) $60 \mathrm{~min}, 500 \times$

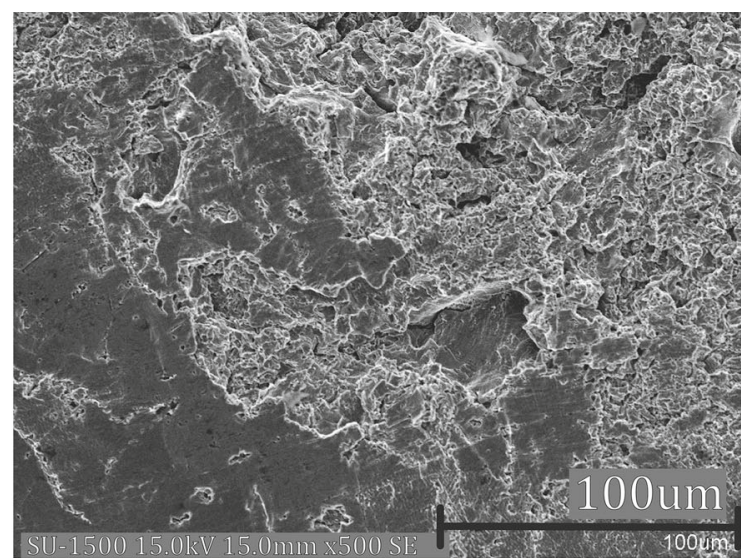

(b) $40 \mathrm{~min}, 500 \times$

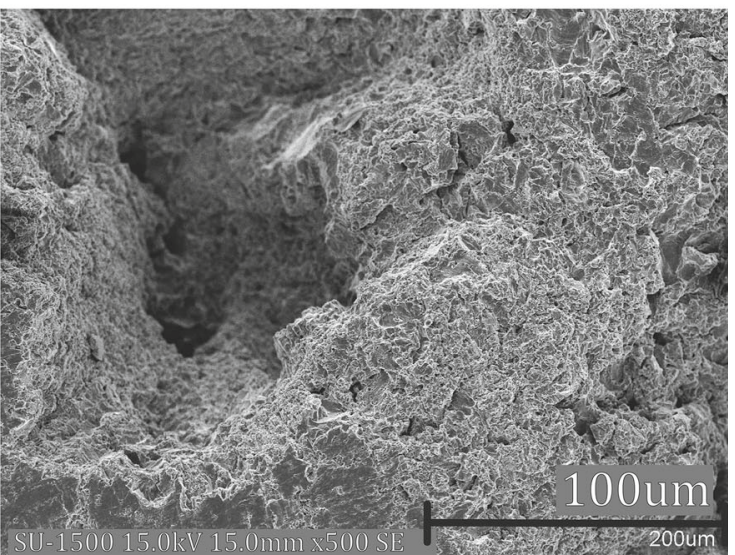

(d) $80 \min , 250 \times$

Fig. 15 SEM pictures of surface of SLM625P as a function of erosion time

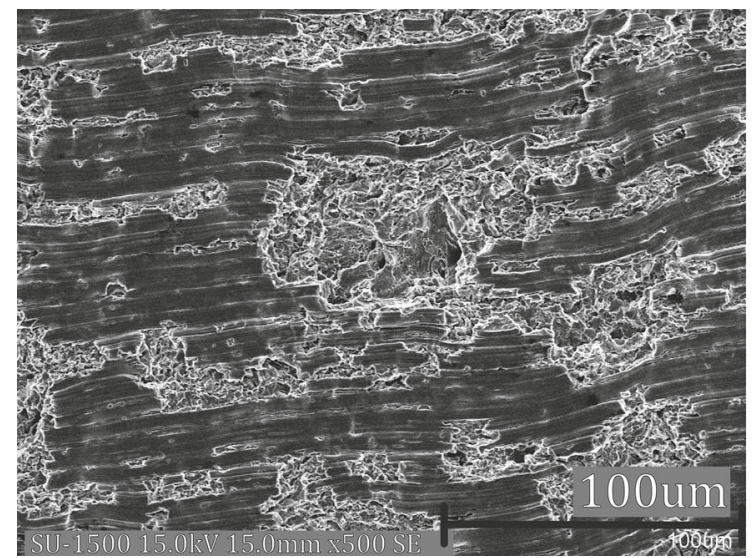

(a) $20 \min , 500 \times$

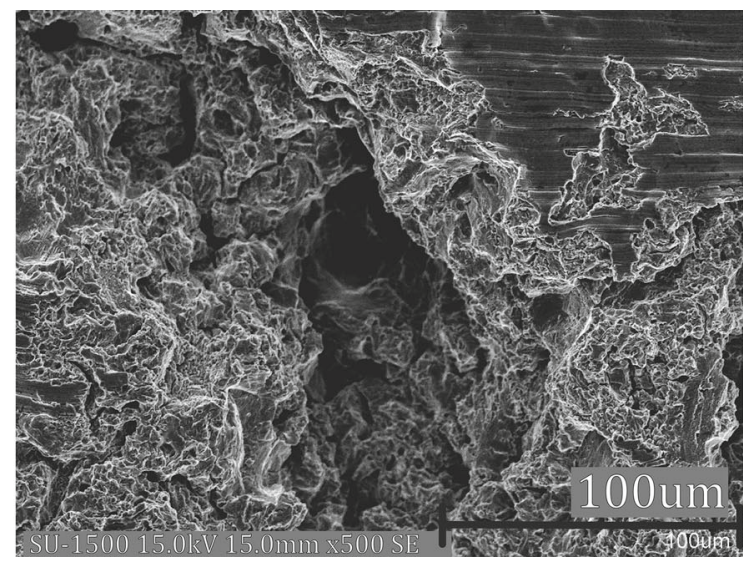

(b) $40 \mathrm{~min}, 500 \times$

Fig. 16 SEM pictures of surface of SLM625 as a function of erosion time 


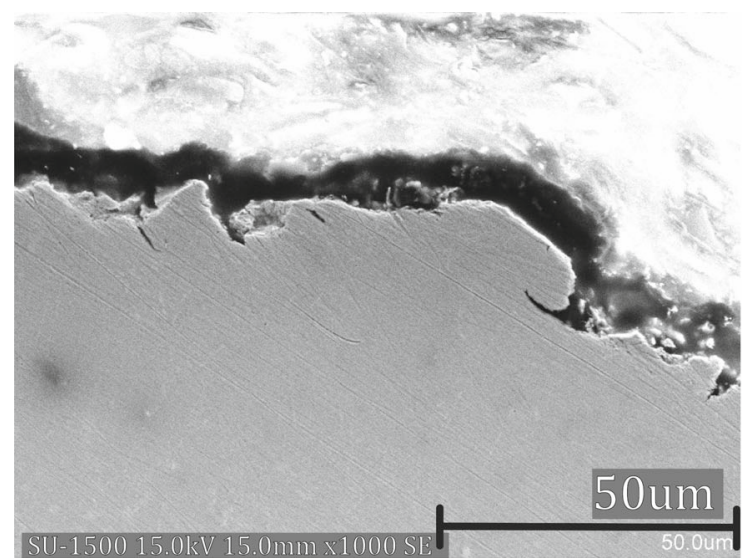

(a) Inco625P, 1000x

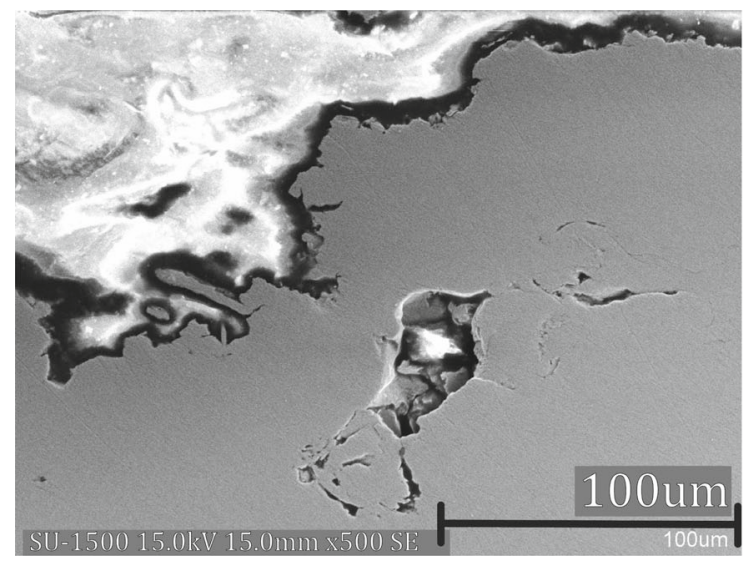

(c) SLM718P, $500 \times$

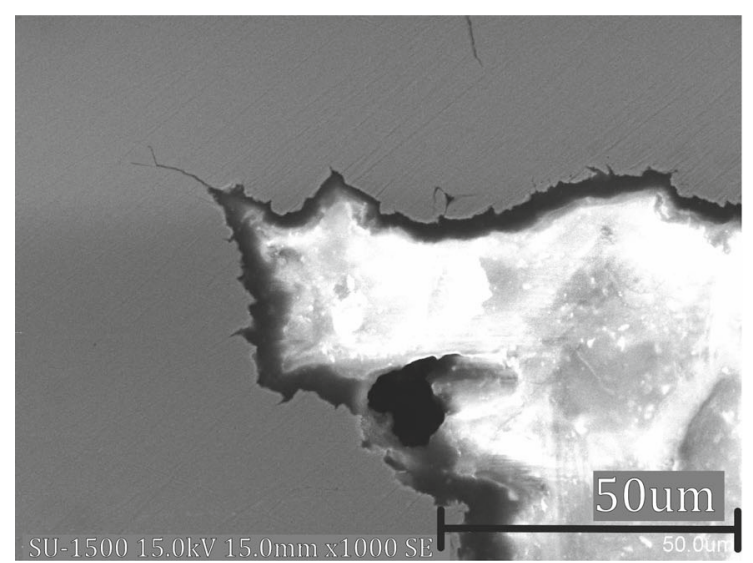

(e) SLM625P, 500×

Fig. 17 Cross-sections of materials after 5 hour of erosion

\section{Conclusion}

The cavitation erosion behavior of Inconel 718 and 625 manufactured by forging and SLM was studied using a standard ASTM G134 cavitation erosion jet. These AM superalloys were previously observed to be eroded when used to transport cryogenic fluids in a rocket engine.

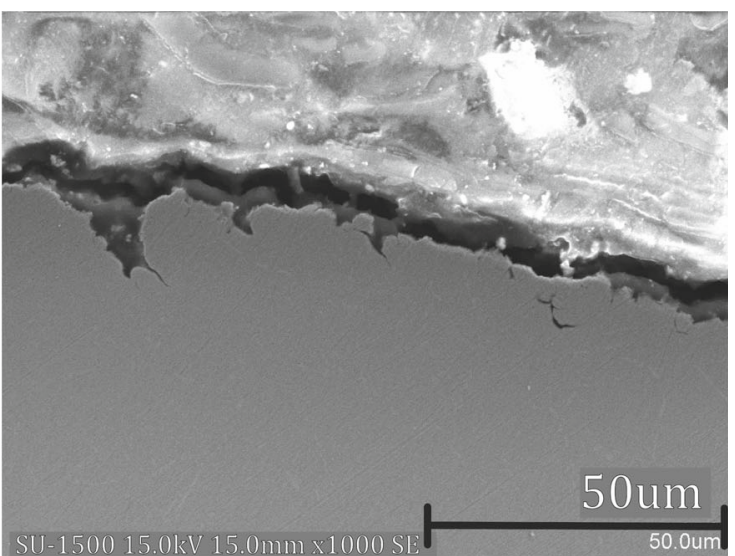

(b) Inco718P, 1000x

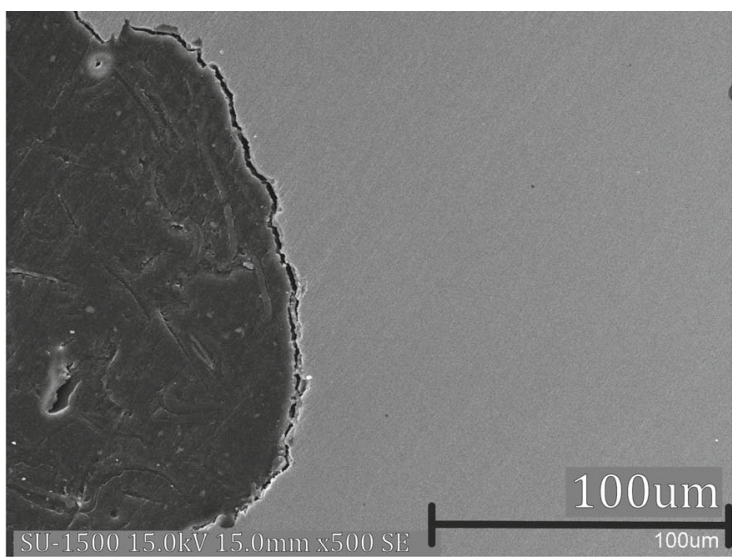

(d) SLM718P, 1000x

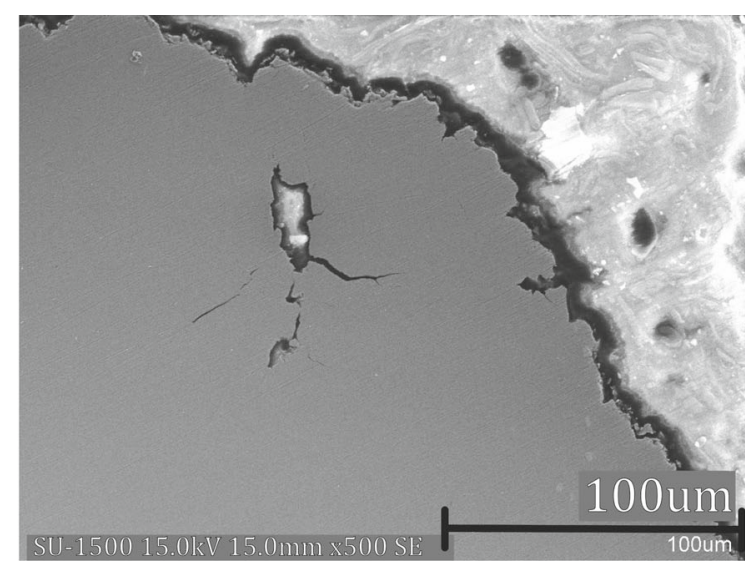

(f) $\mathrm{SLM} 625 \mathrm{P}, 500 \times$

1. The most resistant samples were the machined AM samples SLM718LRa and forged Inco625.

2. Simple machining increased erosion resistance of SLM superalloys up to conventional manufacturing levels in the case of Inconel 718. 
3. Polished samples' erosion resistance was always lower than machined counterparts. This can be explained by the removal of a hardened surface layer.

4. In general, materials with longer erosion periods have lower maximal erosion rates. SLM718 is a notable exception, with the lower incubation period among all studied materials.

5. Harder superalloys tend to have stronger cavitation erosion resistance.

In short, simple machining increases cavitation erosion resistance of superalloys manufactured by SLM, up to and maybe even slightly above their forged counterparts.

Acknowledgments The authors would like to thank the Waseda Research Institute for Science and Engineering (WISE) for providing support to the presented research, in context of the project: "High performance and high reliability research for hydraulic turbomachinery systems". The authors would like to thank the contribution of Mitsubishi Heavy Industries (MHI). We wish specifically thank Mr. Tatsuya Kimura and Mr. Yoshinori Sakurai. The authors would like to thank the contribution of the Japan Aerospace Exploration Agency (JAXA) to this project. We wish specifically thank Mr. Hideo Kawashima.

\section{References}

1. Air Traffic By the Numbers. Tech. rep., Federal Aviation Administration (2019)

2. S.S. Sandhu, A. Shahi, Metallurgical, Wear and Fatigue Performance of Inconel 625 Weld Claddings. J. Mater. Process. Technol. 233, 1-8 (2016)

3. The Annual Compendium of Commercial Space Transportation: 2018. Tech. rep., Federal Aviation Administration (2018)

4. Y. Gao, D. Zhang, M. Cao, R. Chen, Z. Feng, R. Poprawe, J.H. Schleifenbaum, S. Ziegler, Effect of ${ }_{\delta}$ Phase on High Temperature Mechanical Performances of Inconel 718 Fabricated with slm Process. Mater. Sci. Eng. A. 767, 138327 (2019)

5. P. Nandwana, A.M. Elliott, D. Siddel, A. Merriman, W.H. Peter, S.S. Babu, Powderbed Binder Jet 3D Printing of Inconel 718: Densification, Microstructural Evolution and Challenges. Curr. Opin. Solid State Mater. Sci. 21(4), 207-218 (2017)

6. J. Pelleg, Mechanical Properties of Materials. (Springer, New York, 2012)

7. A.A. Popovich, V.S. Sufiiarov, I.A. Polozov, E.V. Borisov, Microstructure and Mechanical Properties of Inconel 718 Produced by slm and Subsequent Heat Treatment. Key Eng. Mater. 651, 665-670 (2015)

8. I. Gibson, D.W. Rosen, B. Stucker et al., Additive Manufacturing Technologies. (Springer, New York, 2014)

9. T. DebRoy, H. Wei, J. Zuback, T. Mukherjee, J. Elmer, J. Milewski, A.M. Beese, A. Wilson-Heid, A. De, W. Zhang, Additive Manufacturing of Metallic Components- Process, Structure and Properties. Prog. Mater. Sci. 92, 112-224 (2018)

10. G.H. Gessinger, Powder Metallurgy of Superalloys: Butterworths Monographs in Materials. (Elsevier, Amsterdam, 2013)

11. Q. Jia, D. Gu, Selective Laser Melting Additive Manufacturing of INCONEL 718 Superalloyparts: Densification, Microstructure and Properties. J. Alloy. Compd. 585, 713-721 (2014)

12. C. Lin, K. Chen, J. He, The Cavitation Erosion Behavior of Electroless Ni-P-SiC Composite Coating. Wear. 261(11-12), 1390-1396 (2006)
13. D. Herzog, V. Seyda, E. Wycisk, C. Emmelmann, Additive Manufacturing of Metals. Acta Mater. 117, 371-392 (2016)

14. H. Hu, Y. Zheng, C. Qin, Comparison of Inconel 625 and Inconel 600 in Resistanceto Cavitation Erosion and Jet Impingement Erosion. Nucl. Eng. Des. 240(10), 2721-2730 (2010)

15. C. Ramesh, D. Devaraj, R. Keshavamurthy, B. Sridhar, Slurry Erosive Wear Behaviour of Thermally Sprayed Inconel-718 Coatings by Aps Process. Wear. 271(9-10), 1365-1371 (2011)

16. P. Suau-Sanchez, A. Voltes-Dorta, N. Cugueró-Escofet, An early Assessment of the Impact of Covid-19 on Air Transport: Just Another Crisis or the End of Aviation as We Know It? J. Transp. Geogr. 86, 102749 (2020)

17. T. Trosch, J. Strößner, R. Völkl, U. Glatzel, Microstructure and Mechanical Properties of Selective Laser Melted Inconel 718 Compared to Forging and Casting. Mater. Lett. 164, 428-431 (2016)

18. T.M. Pollock, S. Tin, Nickel-Based Superalloys for Advanced Turbine Engines: Chemistry, Microstructure and Properties. J. Propul. Power. 22(2), 361-374 (2006)

19. A. Pineau, S.D. Antolovich, High Temperature Fatigue of NickelBase Superalloys-a Review with Special Emphasis on Deformation Modes and Oxidation. Eng. Fail. Anal. 16(8), 2668-2697 (2009)

20. E. Hosseini, V. Popovich, A Review of Mechanical Properties of Additively Manufacturedinconel 718. Addit. Manuf. 30, 100877 (2019)

21. Y. Lu, S. Wu, Y. Gan, T. Huang, C. Yang, L. Junjie, J. Lin, Study on the Microstructure, Mechanical Property and Residual Stress of slm Inconel-718 Alloy Manufactured by Differing Island Scanning Strategy. Opt. Laser Technol. 75, 197-206 (2015)

22. Donachie, M.J., Donachie, S.J.: Superalloys: A Technical Guide. ASM international (2002)

23. S. Zafar, A. Bansal, A. Sharma, N. Arora, C. Ramesh, Dry Erosion Wear Performance of Inconel 718 Microwave Clad. Surf. Eng. 30(11), 852-859 (2014)

24. Z. Baicheng, L. Xiaohua, B. Jiaming, G. Junfeng, W. Pan, S. Chen-nan, N. Muiling, Q. Guojun, W. Jun, Study of Selective Laser Melting (slm) Inconel 718 Part Surface Improvement by Electrochemical Polishing. Mater. Des. 116, 531-537 (2017)

25. X. Jiang, N. Overman, C. Smith, K. Ross, Microstructure, Hardness and Cavitation Erosion Resistance of Different Cold Spray Coatings on Stainless Steel 316 for Hydropower Applications. Mater. Today Commun. 25, 101305 (2020)

26. V. Deshpande, A. Needleman, E. Van der Giessen, Discrete Dislocation Modeling Offatigue Crack Propagation. Acta Mater. 50(4), 831-846 (2002)

27. F. Liu, X. Lin, C. Huang, M. Song, G. Yang, J. Chen, W. Huang, The Effect of Laser Scanning Path on Microstructures and Mechanical Properties of Laser Solid Formed Nickel-Base Superalloy Inconel 718. J. Alloy. Compd. 509(13), 4505-4509 (2011)

28. J.O. Milewski, Additive Manufacturing of Metals: From Fundamental Technology Torocket Nozzles, Medical Implants, and Custom Jewelry. (Springer, New York, 2017)

29. G. Cao, T. Sun, C. Wang, X. Li, M. Liu, Z. Zhang, P. Hu, A.M. Russell, R. Schneider, D. Gerthsen et al., Investigations of ${ }_{\gamma}^{\prime},{ }_{\gamma}^{\prime \prime}$ and ${ }_{\delta}$ Precipitates in Heat-Treated Inconel 718 Alloy Fabricated by Selective Laser Melting. Mater. Charact. 136, 398-406 (2018)

30. C.T. Hsiao, A. Jayaprakash, A. Kapahi, J.K. Choi, G.L. Chahine, Modelling of Material Pitting from Cavitation Bubble Collapse. J. Fluid Mech. 755, 142-175 (2014)

31. Z. Yu-Kang, F. Hammitt, Cavitation Erosion Incubation Period. Wear. 86(2), 299-313 (1983)

32. R.C. Reed, The Superalloys: Fundamentals and Applications. (Cambridge University Press, Cambridge, 2008) 
33. K.H. Kim, G. Chahine, J.P. Franc, A. Karimi, Advanced Experimental and Numerical Techniques for Cavitation Erosion Prediction. (Springer, New York, 2014)

34. K. Thirugnanasambantham, S. Natarajan, Degradation Mechanism for High Temperature Erosion in Surface Modified in718 Superalloy. Surf. Eng. 31(1), 24-28 (2015)

35. R. Singh, S. Tiwari, S.K. Mishra, Cavitation Erosion in Hydraulic Turbine Components and Mitigation by Coatings: Current Status and future Needs. J. Mater. Eng. Perform. 21(7), 1539-1551 (2012)

36. D. Deng, R.L. Peng, H. Brodin, J. Moverare, Microstructure and Mechanical Properties of Inconel 718 Produced by Selective Laser Melting: Sample Orientation Dependence and Effects of Post Heat Treatments. Mater. Sci. Eng. A. 713, 294-306 (2018)

37. K. Moussaoui, W. Rubio, M. Mousseigne, T. Sultan, F. Rezai, Effects of Selectivelaser Melting Additive Manufacturing Parameters of Inconel 718 on Porosity, Microstructure and Mechanical Properties. Mater. Sci. Eng. A. 735, 182-190 (2018)

38. D. Zhang, W. Niu, X. Cao, Z. Liu, Effect of Standard Heat Treatment on the Microstructure and Mechanical Properties of
Selective Laser Melting Manufactured Inconel 718 Superalloy. Mater. Sci. Eng., A. 644, 32-40 (2015)

39. S. Suresh, Fatigue of Materials. (Cambridge University Press, Cambridge, 1998)

40. I. Koutiri, E. Pessard, P. Peyre, O. Amlou, T. De Terris, Influence of slm Process Parameters on the Surface Finish, Porosity Rate and Fatigue Behavior of As-Built Inconel 625 Parts. J. Mater. Process. Technol. 255, 536-546 (2018)

41. J.R. Zhao, F.Y. Hung, T.S. Lui, Erosion Resistance and Particle Erosion-Induced Tensile Embrittlement of 3d-Selective Laser Melting Inconel 718 Superalloy. Metals. 10(1), 21 (2020)

42. X. Zhao, J. Chen, X. Lin, W. Huang, Study on Microstructure and Mechanical Properties of Laser Rapid Forming Inconel 718. Mater. Sci. Eng. A. 478(1-2), 119-124 (2008)

Publisher's Note Springer Nature remains neutral with regard to jurisdictional claims in published maps and institutional affiliations. 